\title{
POTENTIELS ET LIMITES DE L’ANALYSE POLLINIQUE DE SPÉLÉOTHÈMES QUATERNAIRES : APPLICATIONS À LA RECONSTITUTION DE L'ENVIRONNEMENT VÉGÉTAL DE L'HOMME PRÉHISTORIQUE SUR LE POURTOUR NORD-MÉDITERRANÉEN
}

\author{
Vincent LEBRETON, Anne-Sophie LARTIGOT, Elena KARATSORI, \\ Erwan MESSAGER, Laurent MARQUER \& Josette RENAULT-MISKOVSKY
}

\begin{abstract}
RÉSUMÉ
En contexte archéologique, l'analyse pollinique de spéléothèmes offre l'opportunité de caractériser la composition de l'environnement végétal de l'Homme préhistorique. L'évaluation des processus taphonomiques affectant la pluie pollinique lors du dépôt dans la calcite souligne les limites et les potentiels de ce type de support. Les résultats obtenus sur des sites préhistoriques du pourtour nord-méditerranéen sont confrontés et corrélés à l'ensemble de l'information paléoécologique livrée par les données multidisciplinaires. Ces études palynologiques tentent aussi d'estimer la distribution spatiale des écosystèmes végétaux, la composante locale étant souvent la plus significative. Quand la formation des spéléothèmes coïncide avec des niveaux archéologiques, l'environnement local de l'Homme préhistorique peut être précisé.
\end{abstract}

Mots-clés : Palynologie, Spéléothème, Taphonomie, Paléoenvironnement, Quaternaire, Préhistoire.

\section{ABSTRACT}

POTENTIALS AND LIMITS OF POLLEN ANALYSIS ON QUATERNARY SPELEOTHEMS: AN ATTEMPT TO RECONSTRUCT THE VEGETAL ENVIRONMENT OF PREHISTORIC HUMAN IN THE NORTHERN MEDITERRANEAN.

Pollen analysis on speleothems carry out detailed vegetal environment data in archaeological context. Pollen rain recorded in calcite is subject to a variety of taphonomic processes, pointing out limits and potentials of pollen analyses on speleothems. These records are evaluated in the light of multidisciplinary palaeoecological information from Northern Mediterranean prehistoric settlements. A main contribution of local vegetation with regard to spatial distribution of the different vegetal ecosystems is observed. When speleothem growths and archaeological layer deposits are synchronous, local environment of the prehistoric populations can be accurately documented.

Key-words : Palynology, Speleothem, Taphonomy, Palaeoenvironment, Quaternary, Prehistory.

\section{1 - INTRODUCTION}

Depuis les travaux de Von Post (1916), les analyses palynologiques se sont développées progressivement en multipliant les études sur des supports sédimentaires diversifiés. Les dépôts fossiles constitués d'accumulation de végétaux firent très tôt l'objet d'investigations sporo-polliniques, comme les sédiments paléozoïques réservoirs de pétroles et les charbons (Dijkstra, 1946), ainsi que les tourbes post-glaciaires (Van Campo, 1950). Alors que ces sédiments étaient particulièrement propices à la réception et à la conservation des spores et grains de pollen, les sédiments lacustres, marins, fluviatiles, lœssiques, archéologiques, de nature plus minérale, nécessitaient des innovations successives pour améliorer le rendement d'extraction du matériel sporo-pollinique (Sittler, 1955 ; Van Campo \& Leroi-Gourhan, 1956 ; Delcourt et al., 1959 ; Frenzel, 1964 ; Girard \& Renault-Miskovsky, 1969 ; Bastin, 1971 ; Juvigné, 1973). Ces méthodes, particulièrement performantes, amenèrent aussi à s'intéresser au contenu pollinique des spéléothèmes quaternaires découverts sur des sites préhistoriques (Bastin, 1978 ; Renault-Miskovsky \& Girard, 1978).

Les spéléothèmes désignent l'ensemble des formations minérales carbonatées pouvant être construites

Département de Préhistoire du Muséum national d'Histoire naturelle, UMR 5198 du CNRS, Institut de Paléontologie Humaine, 1 rue René Panhard, 75013 Paris.

E-mails : lebreton@mnhn.fr / as1@mnhn.fr / elenak@freesurf.fr / erwan.m@mnhn.fr / marquer@mnhn.fr / jrm@mnhn.fr 
par des empilements de lamines en milieu karstique. Ils se constituent suite à l'écoulement de solutions minéralisées sur les parois calcaires de l'encaissant. Parmi les spéléothèmes, les stalactites, stalagmites, planchers stalagmitiques et draperies se prêtent à l'analyse palynologique. Par ailleurs, les méthodes de datations sur ces formations carbonatées placent dans un cadre chronologique les interprétations paléoécologiques (Harmon et al., 1975 ; Atkinson et al., 1978; Li et al., 1989).

Cet article est consacré à l' analyse palynologique de spéléothèmes de neuf sites préhistoriques localisés sur le pourtour nord-méditerranéen, entre la France méridionale et le sud de la Turquie (fig. 1). Dans ce cadre géographique, les enregistrements sédimentaires quaternaires continentaux propices à la conservation des grains de pollen sont rares (Wijmstra, 1969 ; Wijmstra \& Smit, 1979 ; Follieri et al., 1988 ; Pons \& Reille, 1988 ; Tzedakis, 1993, 1999, Tzedakis et al., 2006 ; Allen et al., 2000). Les gisements suivants ont pour caractéristique commune de présenter une diversité de spéléothèmes en relation stratigraphique avec des dépôts archéologiques quaternaires. Le Vallonnet, à ce jour plus vieil habitat en grotte (Lumley, 1988; Yokoyama et al., 1988), comporte cinq ensembles stratigraphiques, dont deux spéléothèmes. Le remplissage de La Basura est essentiellement composé de spéléothèmes de plus de deux mètres d'épaisseur, enregistrant le contexte paléoenvironnemental et paléoclimatique depuis le début du Pléistocène moyen (Chiapella, 1955 ; Molleson et al., 1972 ; Délibrias, 1985 ; Yokoyama et al., 1985 ; Rousseau et al., 2006). Sur le site de L'Arago, plusieurs planchers stalagmitiques s'insèrent dans la stratigraphie des sédiments archéologiques ayant livré les restes d'Homo erectus (Lumley, 1981 ; Yokoyama et al., 1982 ; Lumley et al., 1984 ; Shen, 1985 ; Falguères et al., 2004). Dans la grotte de Karaïn E, la séquence stratigraphique présente une alternance de dépôts détritiques et d'horizons concrétionnés où s'intercalent différentes formations stalagmitiques (Otte et al., 1998 ; Yalçinkaya, 1998 ; Farkh, 2004). La phosphatisation du remplissage détritique de la Baume Bonne, qui a altéré le matériel organique (Perrenoud, 1993 ; Gagnepain \& Gaillard, 1996 ; Mestour, 1996), n'a pas affecté les spéléothèmes (Falguères et al., 1991). Au Lazaret, le remplissage incluant de nombreuses unités archéostratigraphiques est recouvert de spéléothèmes (Lumley, 1969 ; Lumley-Woodyear, 1970 ; Lumley et al., 1976 ; Shen, 1985). A Kalamakia, de nombreuses stalagmites, dont un important pilier, sont contemporaines des niveaux archéologiques (Lumley \& Darlas, 1994 ; Voinchet \& Darlas, 1998 ; Lecervoisier, 2003). Le remplissage de l'abri Pié-Lombard, reposant sur un plancher stalagmitique, contient du matériel archéologique associé à des restes néandertaliens, ainsi que des fragments de stalactites détachés du plafond (Texier, 1974 ; Valladas et al., 1987, 1988). A Caverna delle Fate, quatre planchers stalagmitiques se sont formés dans la partie supérieure du remplissage contenant des restes néandertaliens associés à des vestiges archéologiques (Echassoux et al., 1983 ; Giacobini et al., 1984 ; Falguères et al., 1990). Le tableau 1 rappelle la localisation et le contexte environnemental actuel de ces gisements en précisant les données culturelles et paléoanthropologiques dans le cadre chronologique du Quaternaire. La place des spéléothèmes analysés et les dates associées sont portées en regard.

L'enregistrement pollinique en milieu karstique, et notamment dans les spéléothèmes, est un phénomène complexe dans lequel interviennent de multiples facteurs géologiques, atmosphériques et biologiques. Après rappels des différents paramètres définissant la taphonomie pollinique, les limites et potentiels de l'analyse palynologique des spéléothèmes sont présentés. Cette démarche méthodologique s'appuie sur les principaux résultats obtenus à partir des sites sélectionnés pour cette étude. Quand les résultats issus de ces

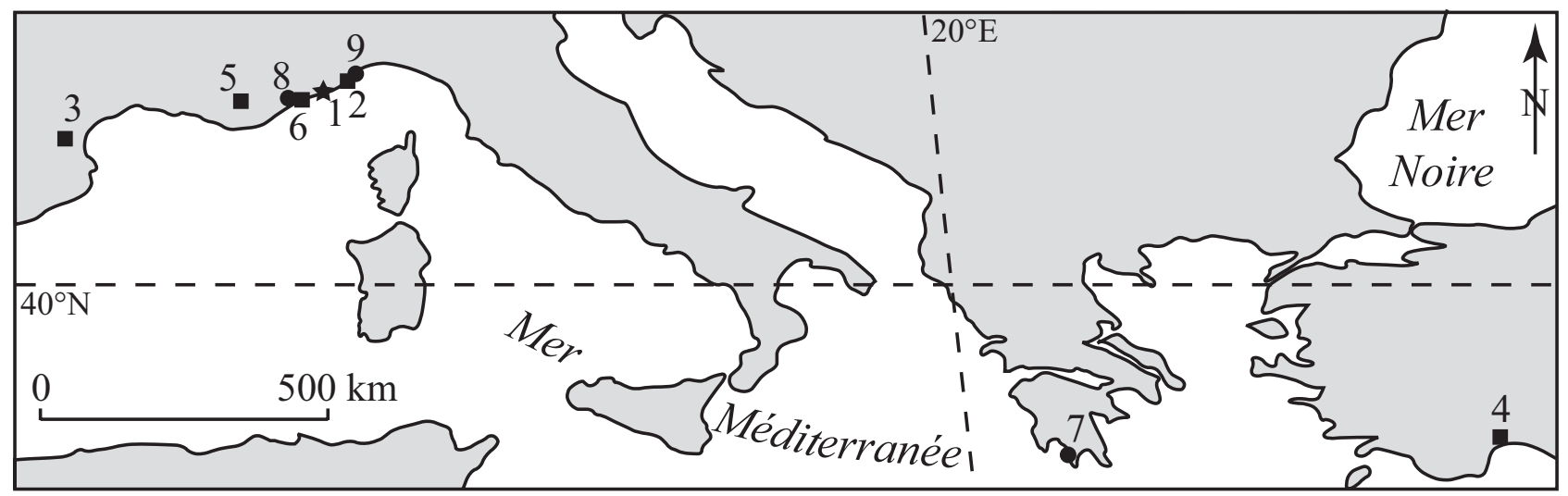

Fig. 1 : Localisation des sites préhistoriques présentés dans le texte (du plus ancien au plus récent). $\star$ Pléistocène inférieur : 1. Le Vallonnet (Roquebrune-Cap-Martin, France) ; • Pléistocène moyen : 2. La Basura (Toirano, Italie), 3. L’Arago (Tautavel, France), 4. Karaïn E (Yagca, Turquie), 5. La Baume Bonne (Quinson, France), 6. Le Lazaret (Nice, France) ; • Pléistocène supérieur : 7. Kalamakia (Aréopolis, Grèce), 8. Pié-Lombard (Tourettes-sur-Loup, France), 9. Caverna delle Fate (Finale Ligure, Italie).

Fig. 1: Location of the prehistoric settlements introduced in the text (from the oldest to the most recent). $\star$ Early Pleistocene: 1. Le Vallonnet (Roquebrune-Cap-Martin, France); Middle Pleistocene: 2. La Basura (Toirano, Italy), 3. L'Arago (Tautavel, France), 4. Karä̈ E (Yagca, Turkey), 5. La Baume Bonne (Quinson, France), 6. Le Lazaret (Nice, France); - Upper Pleistocene: 7. Kalamakia (Aréopolis, Greece), 8. Pié-Lombard (Tourettes-surLoup, France), 9. Caverna delle Fate (Finale Ligure, Italy). 


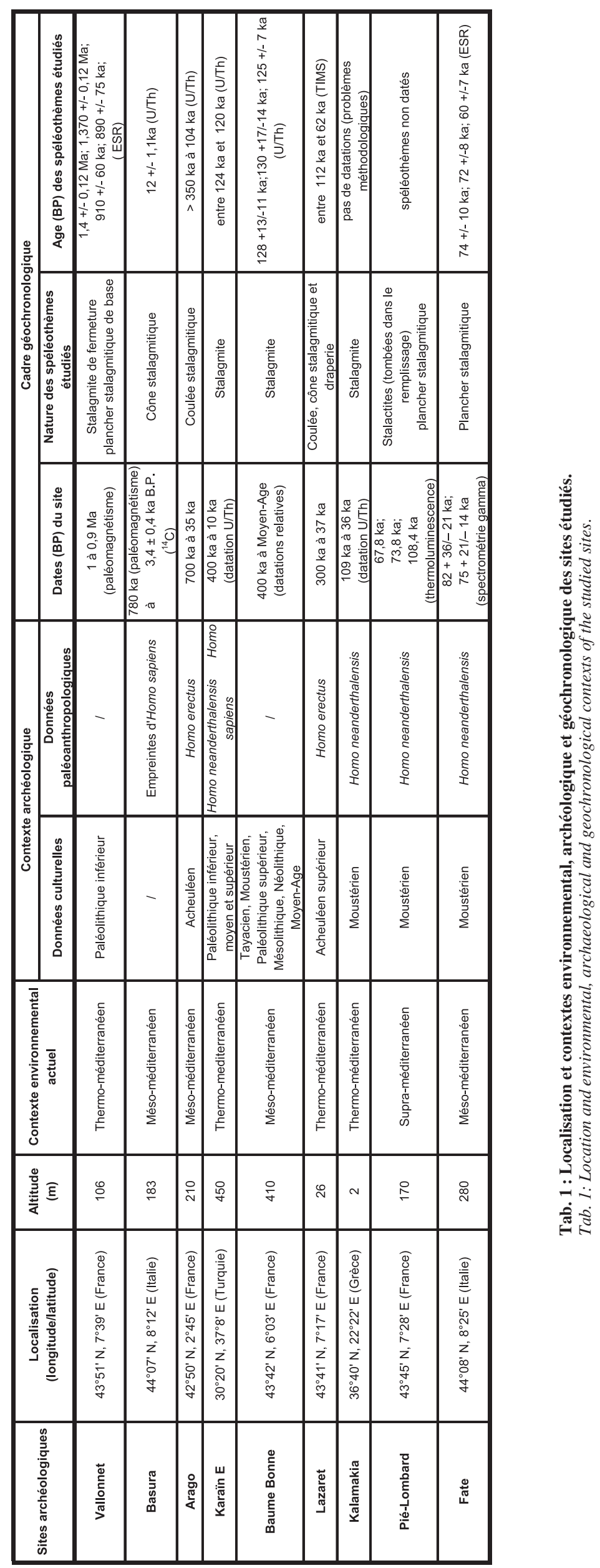


analyses peuvent être corrélés avec les niveaux archéologiques, il devient possible de décrire l'environnement végétal de l'Homme préhistorique.

\section{2 - MATÉRIEL ET MÉTHODE}

\section{1 - ÉCHANTILLONNAGE}

Les prélèvements sur chacun des sites sont conduits selon la nature du spéléothème. Le volume, la hauteur ou encore la disposition de la formation carbonatée dans la cavité amènent à recourir à des équipements différents. Une scie à disque électrique (meuleuse) a été utilisée pour découper les spéléothèmes au Vallonnet, à la Baume Bonne, à Karaïn E, au Lazaret, à Kalamakia et à Fate. Un équipement plus lourd avec carottier à tête diamantée a été installé pour prélever des carottes à l'Arago (diamètre $8 \mathrm{~cm}$ ) et à la Basura (diamètre $4 \mathrm{~cm}$ ). A Pié-Lombard, trois carottiers de diamètres croissants, montés sur une foreuse électrique fixe, ont permis d'extraire le cœur d'une stalactite puis deux anneaux concentriques. Un autre spéléothème (fragment de stalactite détaché) a été prélevé dans le remplissage archéologique.

Au laboratoire, l'échantillon est dessiné avant d'être débité. Les prélèvements se font en suivant les lacunes de sédimentation formant des zones de rupture naturelle et/ou, quand visible, en débitant des ensembles de lamines distingués par colorimétrie (fig. 2).

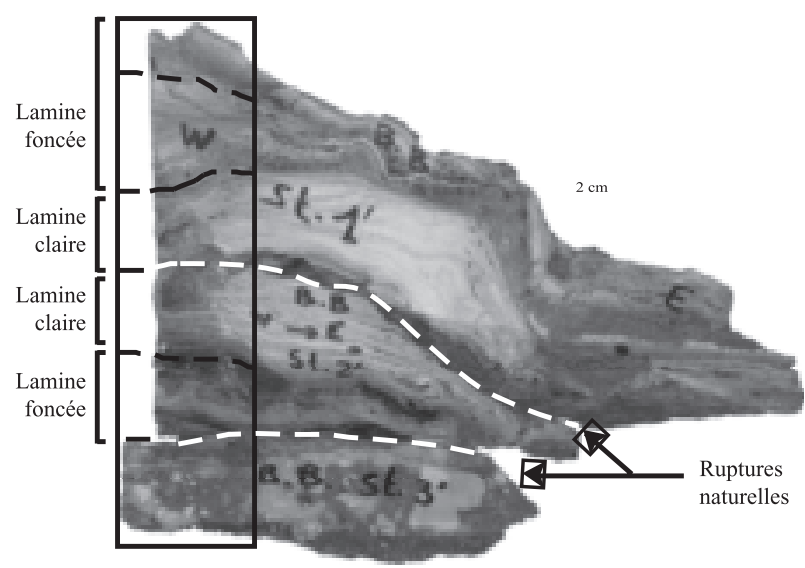

Fig. 2 : Échantillonnage palynologique sur spéléothème (La Baume Bonne).

Fig. 2: Palynological sampling on speleothem (Baume Bonne).

\section{2 - PROTOCOLE D’EXTRACTION}

L'extraction chimique du matériel sporo-pollinique des échantillons prélevés sur des spéléothèmes diffère sensiblement du protocole classique initialement décrit par Sittler (1955) : HCl, HF, KOH, flottation en liqueur dense. Le poids traité est variable, dépendant essentiellement du volume disponible lors de l'échantillonnage. Une masse importante va permettre de compenser la concentration sporo-pollinique potentiellement faible de ce type de sédiment. Dans ce cas, la phase initiale d'attaque à $\mathrm{HCl}$ est longue, afin de dissoudre la fraction minérale carbonatée dominante.

\section{3 - TAPHONOMIE POLLINIQUE DANS LES SPÉLÉOTHÈMES}

Entre l'émission des grains de pollen par la végétation et l'identification de la pluie pollinique fossile lors de l'analyse, certains processus physico-chimiques et biologiques sont susceptibles de modifier le signal. Ils sont regroupés sous l'appellation de taphonomie pollinique. Pour identifier l'ensemble de ces phénomènes, il faut considérer l'origine des grains de pollen avant le dépôt, leurs conditions de sédimentation lors de la précipitation des carbonates et leur conservation dans la calcite après le dépôt.

\section{1 - ORIGINE DES GRAINS DE POLLEN RETROUVÉS DANS LES SPÉLÉOTHÈMES}

Le dépôt du matériel sporo-pollinique enregistré dans les spéléothèmes provient de plusieurs sources possibles (fig. 3).

Le pollen peut être transporté par les eaux du karst, en circulant depuis le plateau à travers les fissures du réseau ouvert dans le massif calcaire (Smart \& Friederich, 1986 ; Baker et al., 1997). Ces fracturations et cavités du réseau karstique peuvent engendrer une rétention du flux pollinique (Bui-Thi-Mai \& Girard, 1988 ; Coles et al., 1989 ; Burney \& Burney, 1993), d'autant plus importante que la végétation est dense et le sol bien développé au-dessus de la cavité (McGarry $\&$ Caseldine, 2004).

Le matériel sporo-pollinique dans les spéléothèmes peut aussi provenir du transport par les circulations d'air au sein de la cavité (Coles et al., 1989 ; Carrasco et al., 1995 ; McGarry \& Caseldine, 2004 ; Fairchild et al., 2006). Ces mouvements dépendent entre autres de la taille et de l'orientation de la grotte, ainsi que du nombre d'entrées (Atkinson, 1981 ; Smithson, 1982). L'importance quantitative des apports polliniques éoliens est aussi inversement proportionnelle à la distance entre l'entrée de la grotte et le spéléothème (Van Campo \& Leroi-Gourhan, 1956; Smithson, 1982; Burney \& Burney, 1993 ; Coles \& Gilberston, 1994).

L'action des êtres vivants, végétaux ou animaux, est également à considérer (Van Campo \& Leroi-Gourhan, 1956 ; Coles et al., 1989). Ainsi, les animaux (chiroptères, ours, hyènes, insectes) et/ou les Hommes peuvent transporter fortuitement sur un site préhistorique, du matériel sporo-pollinique de la végétation extérieure (Martin \& Sharrock, 1964; Leroi-Gourhan, 1966, 1981 ; Davis \& Anderson, 1987 ; Bui-Thi-Mai \& Girard, 1988, 2000 ; Diot, 1991 ; Navarro Camacho et al., 2000 ; Carrión et al., 2001, 2005). Ce matériel peut ensuite se trouver incorporé dans une concrétion du site et refléter les déplacements dans l'environnement local. 


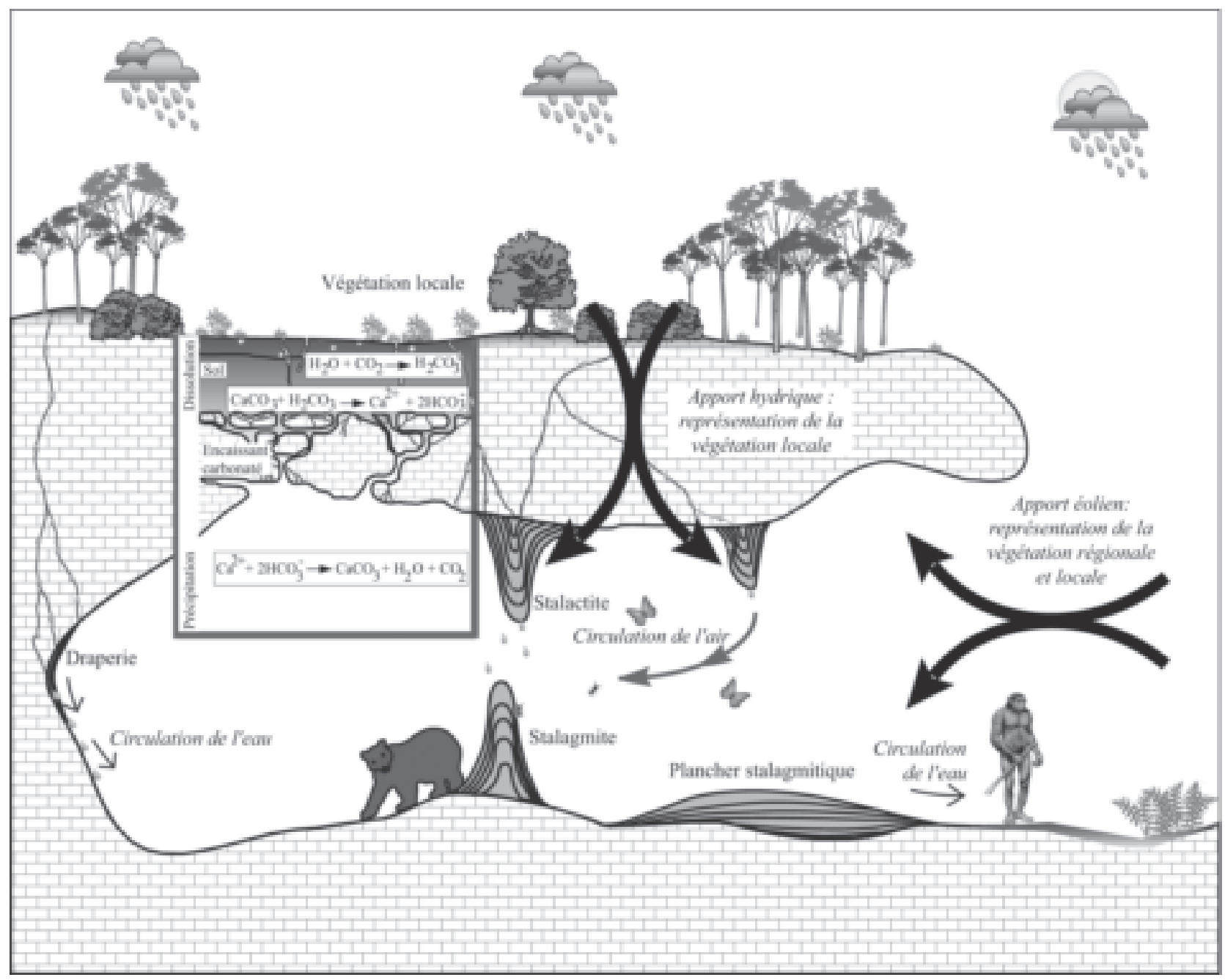

Fig. 3 : Formation et nature des spéléothèmes et origine des apports polliniques (modifié d'après McGarry \& Caseldine, 2004 ; Fairchild et al., 2006).

Fig. 3: Growth and different types of speleothems and possible pollen transport origins (modified from McGarry \& Caseldine, 2004; Fairchild et al., 2006).

Par ailleurs, un porche à l'entrée des grottes peut être favorable à l'installation de plantes hygrophiles et sciaphiles. Les spores et grains de pollen de ces plantes peuvent alors être mieux représentés que le reste de la végétation locale, du fait de la proximité avec le spéléothème étudié (Coles et al., 1989).

\section{3 - FORMATION DES SPÉLÉOTHÈMES ET PIÉGEAGE DU MATÉRIEL SPORO-POLLINIQUE}

Les spéléothèmes se forment dans les réseaux karstiques d'un encaissant calcaire. L'épikarst est recouvert par un sol plus ou moins riche en matière organique. L'eau s'infiltrant depuis le sol dans l'épikarst transporte des éléments minéraux, organiques et biogéochimiques qui se retrouvent ensuite dans les spéléothèmes (Fairchild et al., 2006). Parmi ces éléments, se trouve le matériel sporo-pollinique, des radioéléments, des acides (humiques), de l'oxygène et du dioxyde de carbone, véhiculés par l'eau. Cette eau devient acide et dissout alors le carbonate de calcium de l'encaissant. Elle se charge en bicarbonate de calcium, instable. Les phénomènes d'évaporation et de dégazage de $\mathrm{CO}_{2}$ dans l'eau vont modifier les équilibres chimiques et permettre la précipitation du carbonate de calcium (Holland et al., 1964). Ce sont ces phases de dissolution puis de précipitation qui assurent la formation des spéléothèmes (fig. 3). Lors de la précipitation des carbonates, les conditions de piégeage des différents éléments biogéochimiques dans la matrice du spéléothème forment un système clos et le signal pollinique est très bien conservé.

La vitesse de cristallisation des carbonates de calcium est difficile à appréhender. Selon les auteurs, cette vitesse peut varier d'un facteur 100 , de 0,01 à $1 \mathrm{~mm}$ par an (Baker et al., 1993 ; Baker \& Smart, 1995 ; Blanc, 2005). Au cours de la cristallisation, une fraction sédimentaire détritique du remplissage de la grotte peut se trouver incorporée dans la matrice carbonatée.

\section{3 - CONSERVATION POST-DÉPOSITIONNELLE DU MATÉRIEL SPORO-POLLINIQUE}

L'intérêt de l'analyse palynologique de spéléothème réside dans le fait que la calcite, une fois cristallisée, forme un système stable et clos où tous les éléments 
biogéochimiques, dont le pollen, sont enfermés et conservés (Bastin, 1978; Bui-Thi-Mai \& Girard, 1988 ; Burney \& Burney, 1993). En l'absence d'actions chimiques secondaires, aucun processus taphonomique post-dépositionnel ne s'exerce. Si des processus d'altération interviennent, ils sont facilement identifiables, formant des dépôts pulvérulents rarement analysés en palynologie (Leroi-Gourhan, 1967).

\section{4 - ILLUSTRATION DES LIMITES ET POTENTIELS DE L'ANALYSE POLLINIQUE DE SPÉLÉOTHÈMES QUATERNAIRES}

\section{1 - ÉCHANTILLONNAGE ET ANALYSE DE LA FORMATION DU SPÉLÉOTHÈME}

\subsection{1 - Échantillonnage}

Dans le cas des études paléoclimatiques à partir d'analyses isotopiques développées sur les spéléothèmes, les approches à haute résolution sont courantes (Harmon et al., 1975 ; Atkinson et al., 1978; Li et al., 1989 ; Shopov \& Dermendjiev, 1990 ; Genty \& Quinif, 1996 ; Genty et al., 2003). En effet, la masse d'échantillons requise est minime et les micro-carottiers utilisés permettent des analyses lamine à lamine. Mais en vue d'une analyse pollinique de spéléothème, l'usage de ce type d'équipement n'est pas possible. Du fait de la faible quantité des grains de pollen contenus dans l'eau participant à la précipitation de la calcite, il est nécessaire de traiter une grande quantité de carbonate
(Burney et al., 1994). Cela suppose fréquemment d'englober plusieurs lamines, pouvant conduire à une superposition de différents épisodes climatiques. Il y a alors un risque de lissage de l'information environnementale puisque seuls les événements très tranchés et durables seront saisis (Burney et al., 1994 ; McGarry \& Caseldine, 2004). L'échantillonnage des spéléothèmes à des fins d'analyses palynologiques doit donc être effectué avec attention.

L'échantillonnage entrepris sur les différents spéléothèmes de l'ensemble E du Lazaret, reposant directement sur le remplissage archéologique de la grotte, illustre ce point. Une tranchée a été taillée dans une coulée, ainsi que dans un petit dôme stalagmitique, d'une vingtaine de centimètres d'épaisseur. La calcite de la coulée stalagmitique ne présente aucune lamine et, de ce fait, l'échantillonnage au laboratoire a été réalisé de façon arbitraire (fig. 4). Par contre, le dôme stalagmitique présente de nombreuses lamines de couleurs différentes, allant du beige au rouge-brun. Deux types d'échantillonnage ont été réalisés sur cette stalagmite (fig. 4). La première colonne de prélèvement (échantillonnage 1) suit les zones de rupture apparues lors de la découpe, in situ, ainsi que les gradients de couleurs. Cinq échantillons, d'un poids compris entre 78 et 155 grammes, ont été débités. Sur la même stalagmite, un deuxième découpage (échantillonnage 2) est calé sur les résultats des analyses isotopiques qui corrèlent la croissance du spéléothème avec les sous-stades du stade isotopique marin (MIS) 5 (Rousseau et al., 2005). Dix échantillons, de volume plus réduit et donc de masse moindre que les précédents (entre 10 et

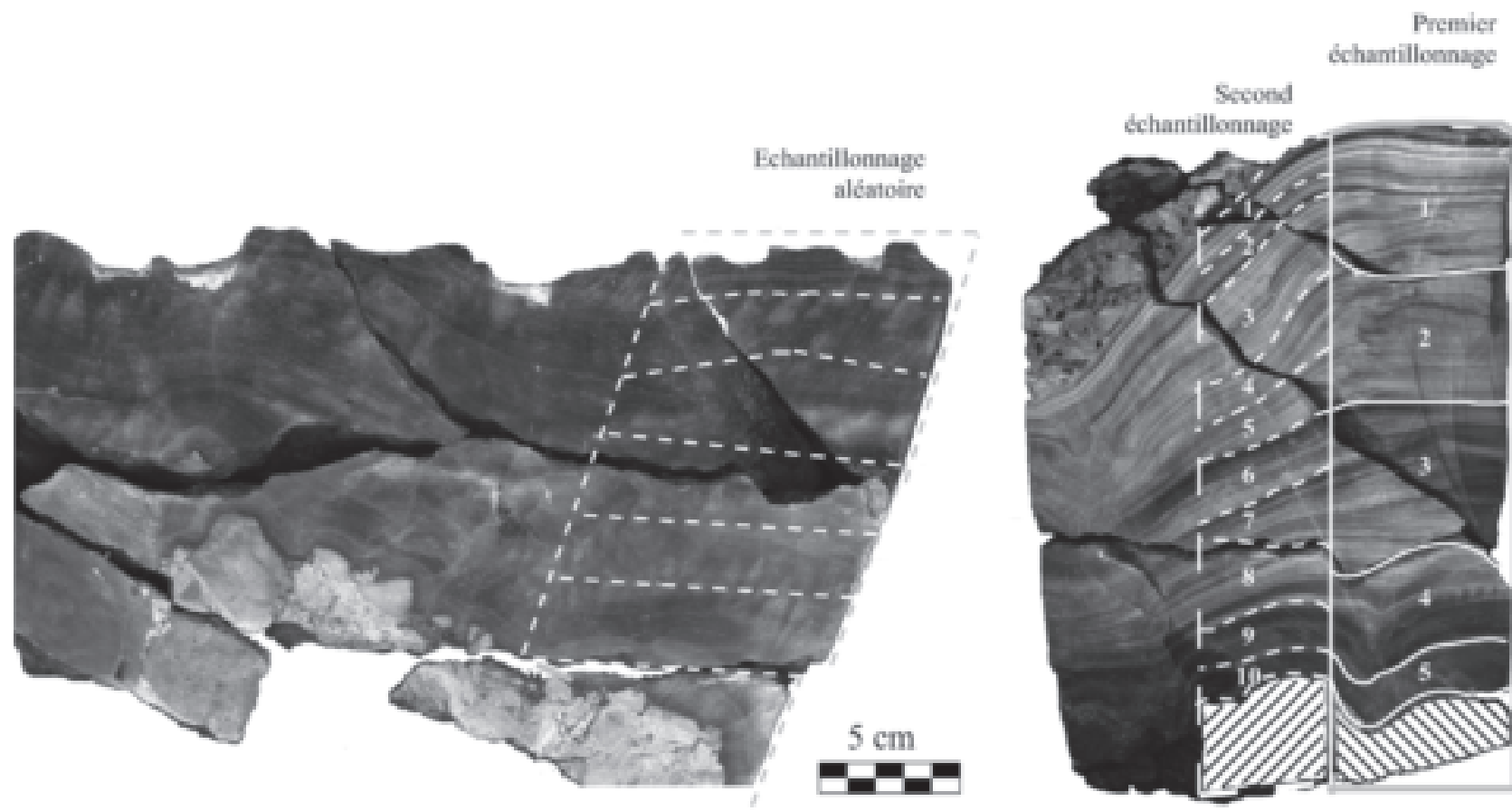

Coulée stalagmitique homogène - Zone G26

Dôme stalagmitique laminaire - Zone N21 (les échantillons de la base du dôme ne sont pas analysables)

Fig. 4 : Échantillonnage des spéléothèmes du Lazaret (clichés A.S. Lartigot)

Fig. 4 : Sampling on speleothems from Le Lazaret (photos A.S. Lartigot). 


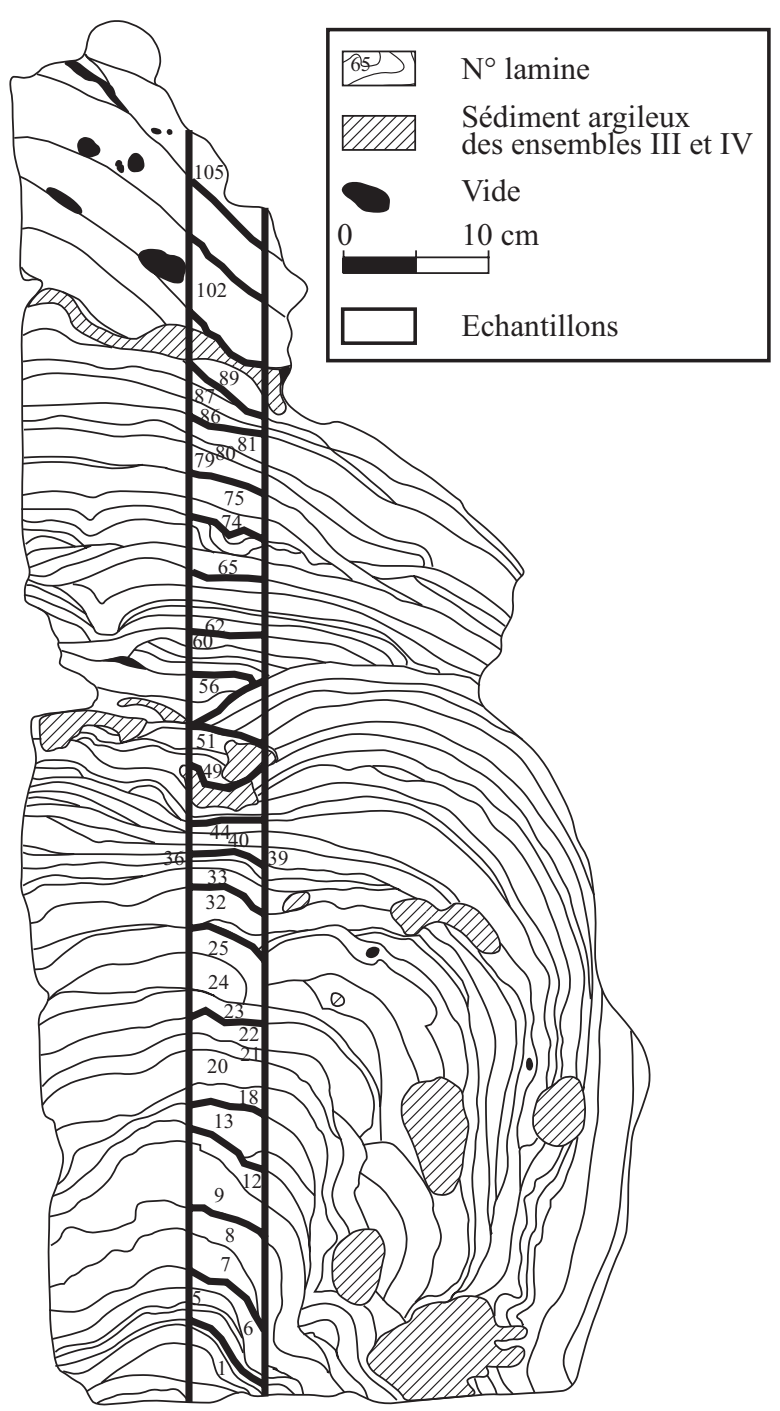

Fig. 5 : Représentation de l'échantillonnage du pilier stalagmitique de Kalamakia (modifié d'après Lecervoisier, 2003).

Fig. 5: Sampling profile on the large stalagmite from Kalamakia (modified from Lecervoisier, 2003).

114 grammes) ont alors été prélevés. Certains fragments des deux débitages se superposent. Les cinq échantillons de la première découpe livrent un nombre de spores et grains de pollen suffisants, mais présentant un contenu homogène. Dans la seconde analyse, des variations ont été mises en évidence, bien qu'une partie des échantillons, moins volumineuse, n'ait pas permis d'obtenir suffisamment de matériel sporo-pollinique. Les spectres issus de la première analyse étaient donc biaisés par un échantillonnage aléatoire, englobant de nombreuses lamines.

L'étroite partie du pilier stalagmitique de Kalamakia disponible pour l'analyse pollinique obligeait, lors de l'échantillonnage, à englober parfois plusieurs lamines quand celles-ci étaient fines (fig. 5). Les plus épaisses permettent de réaliser un échantillon unique. A l'inverse, les lamines trop fines sont réunies en un seul échantillon. Après comptage, les très faibles concentrations sporo-polliniques peuvent nécessiter de regrouper deux échantillons consécutifs afin d'obtenir une somme totale suffisante. Toutefois ces regroupements s'opèrent lorsque les échantillons ne sont pas séparés par une surface naturelle nettement visible pouvant traduire un événement paléoclimatique et quand les deux signaux apparaissent similaires.

Dans tous les cas, il apparaît préférable d'optimiser l'échantillonnage en amont de l'analyse, quitte à regrouper des données de spectres consécutifs en aval du comptage, quand le nombre de sporomorphes n'est pas atteint.

\subsection{2 - Analyse de la formation du spéléothème}

La pureté du carbonate de calcium à analyser doit être considérée, lors de l'échantillonnage d'un spéléothème. En effet, au cours de la précipitation, la stalagmite peut inclure une fraction sédimentaire détritique 《 exogène » dont le contenu pollinique s'ajoute à celui de la calcite (Diot, 1988 ; Genty et al., 2001). Ces deux sources polliniques peuvent ne pas être contemporaines et sont alors difficiles à dissocier.

Ainsi à Kalamakia, la calcite du pilier stalagmitique n'est pas pure. Sa couleur rougeâtre traduit l'incorporation de sédiment issu soit du remplissage, soit de l'environnement local via le karst. L'origine des apports polliniques conservés dans le spéléothème est difficile à déterminer. Les tests palynologiques entrepris sur des échantillons de sédiments meubles ont révélé la quasi-stérilité du remplissage: les rares grains de pollen rencontrés sont très corrodés et ils traduisent une intense dégradation post-dépositionnelle. Toutefois, la pluie pollinique émise lors du dépôt a pu se conserver en se trouvant piégée dans le spéléothème au moment de sa formation, avant toute dégradation ultérieure.

\section{2 - ASSEMBLAGES POLLINIQUES ENREGISTRÉS DANS LES SPÉLÉOTHÈMES}

\subsection{1 - Occurrence du pollen dans les spéléothèmes}

Les sites préhistoriques étudiés ici sont tous en milieu méditerranéen où les sédiments continentaux de remplissages de grottes sont peu propices à la conservation du pollen. En revanche, les spéléothèmes enregistrent et conservent bien la pluie pollinique dans des environnements géologiques peu favorables (Bastin, 1978 ; Bui-Thi-Mai \& Girard, 1988 ; Burney \& Burney, 1993). Dès lors, l'analyse palynologique des spéléothèmes se révèle être une source d'informations paléoécologiques supplémentaire.

Le remplissage de la grotte de la Basura est presque exclusivement composé de spéléothèmes. Une couche d'argiles meubles rougeâtres d'environ un mètre d'épaisseur n'a donné lieu qu'à une seule analyse (Arroba, 1985). Afin de connaître l'évolution des paléoenvironnements de la région, les spéléothèmes sont les seuls autres supports d'études disponibles. Une stalagmite reposant sur des empreintes de Homo sapiens a fait l'objet d'une première étude (Arroba, 1985) et deux colonnes proches (TO 83-8 et TO 83-19) ont été prélevées sur le plancher stalagmitique de 
fermeture pour de nouvelles analyses palynologiques. La colonne TO 83-19 est composée de lamines de calcite beiges et d'un voile sommital grisâtre (fig. 6). Le diagramme montre qu'une végétation semi-fermée, dominée par Quercus t. pedunculatalpubescens accompagné de taxons méditerranéens, régnait aux alentours de la grotte, il y a environ 12000 ans. Le climat pouvait être plus humide que l'actuel.

A la Baume Bonne, le sédiment détritique a subi une phase de phosphatisation qui a détruit l'ensemble du matériel organique. Parmi les spéléothèmes formés sur la surface du remplissage, une stalagmite parfaitement conservée a été prélevée en vue d'une étude palynologique (fig. 7). Elle a permis de mettre en évidence une surreprésentation de Pinus, intégré dans un paysage fermé composé d'essences xériques et méditerranéennes. Le climat devait alors être relativement proche de celui régnant actuellement dans les gorges du Verdon. Ponctuellement, les analyses montrent un net recul des essences méditerranéennes, témoignant d'un assèchement du climat. Ce phénomène se visualise par une rupture naturelle dans le spéléothème témoignant d'un arrêt de précipitation de la calcite.

A la Baume Bonne, Lazaret, Karaïn E, Kalamakia et Fate, les échantillons carbonatés sont les seuls à avoir conservé un contenu pollinique interprétable, venant ainsi palier le manque d'informations directes en provenance du remplissage archéologique.

\subsection{2 - Effet filtrant du karst}

L'assemblage pollinique conservé dans les spéléothèmes peut être un enregistrement biaisé de la végétation développée au-dessus du gisement en raison de l'effet filtrant possible en amont du réseau karstique (McGarry \& Caseldine, 2004). Le pollen, comme les éléments minéraux, circule dans l'épikarst. Une partie du contenu pollinique peut alors se retrouver piégée dans les spéléothèmes en fin de parcours au sein du karst. Mais, des configurations karstiques exceptionnelles peuvent favoriser une circulation des seuls éléments dissous dans l'épikarst alors qu'une rétention totale peut affecter le matériel entraîné depuis la surface. A Pié-Lombard, le cœur de la stalactite s'est formé lorsque la grotte était totalement fermée. Dans cette situation, il n'y a donc pas d'apport éolien et la seule origine pollinique possible ne peut être que le karst. Or, les deux échantillons prélevés au centre de ce spéléothème sont stériles. A l'inverse, l'enveloppe externe, contemporaine de l'ouverture de la grotte, permet d'établir un spectre pollinique, mais dont les apports peuvent être éoliens et/ou karstiques. Sans pouvoir trancher, cet exemple démontre cependant que le réseau karstique peut opérer par intermittence une rétention totale du matériel pollinique.

Quelle que soit l'ampleur de la rétention dans le karst, les concentrations sporo-polliniques observées dans

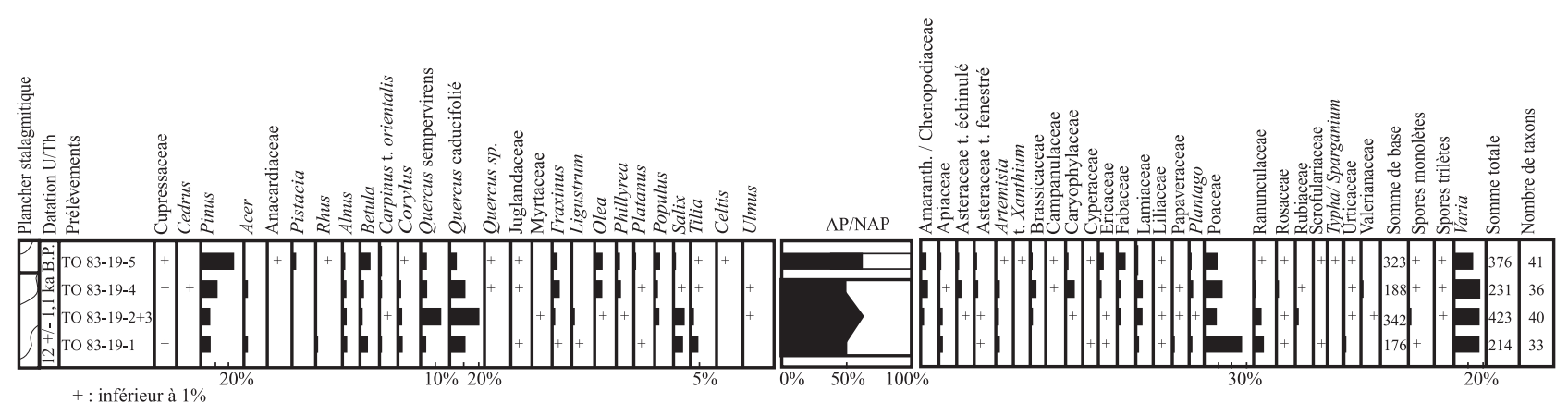

Fig. 6 : Diagramme pollinique détaillé du plancher stalagmitique de fermeture de la Basura (colonne TO 83-19).

Fig. 6: Detailed pollen diagram of the upper stalagmitic floor from Basura (sampling TO 83-19).

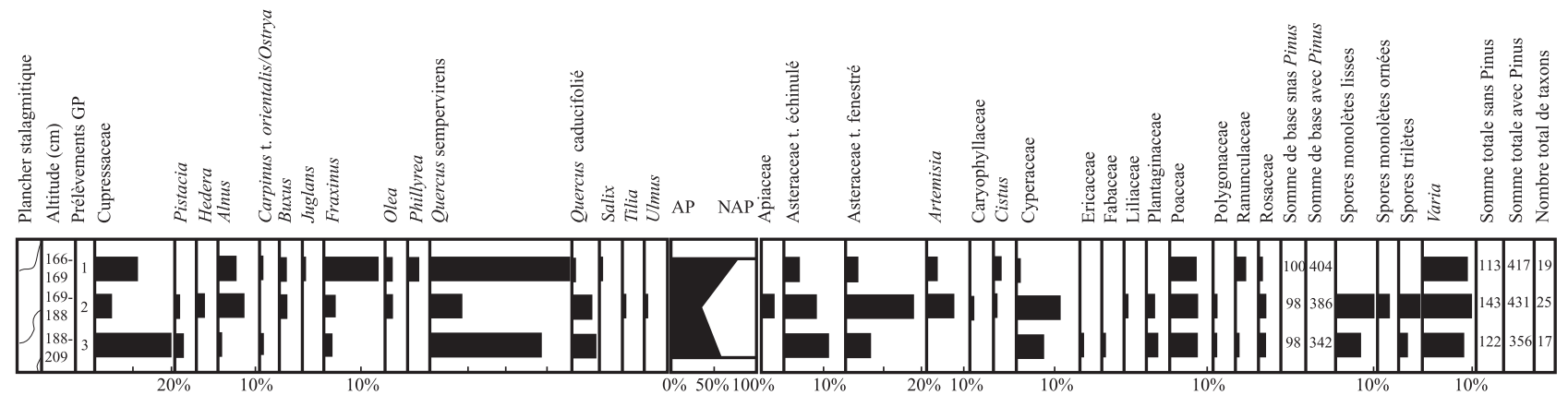

Fig. 7 : Diagramme pollinique détaillé sans Pinus d'une stalagmite de la surface du remplissage de la Baume Bonne.

Fig. 7: Detailed pollen diagram excluding Pinus of an upper stalagmite from Baume Bonne. 
les spéléothèmes sont très basses. A Caverna delle Fate, les concentrations oscillent entre 2 et 19 grains par gramme de sédiment. A Kalamakia, la concentration est même le plus souvent inférieure à 1 grain par gramme de carbonate. Seul dans le dernier échantillon, englobant la lamine la plus externe du pilier stalagmitique, la richesse atteint 88 grains par gramme. Pour Karaïn E, les concentrations sont basses $(\leq 3$ grains par gramme).

\subsection{3 - Validation des spectres polliniques de spéléothèmes}

Le phénomène de filtre que joue le karst s'opère entre le flux pollinique émis dans l'atmosphère et le signal capté dans la calcite. Ceci explique les concentrations faibles rencontrées dans les analyses de spéléothème et la difficulté d'atteindre un comptage de 300 grains par spectre. Toutefois, les assemblages sporo-polliniques conservés dans la calcite ne sont que rarement affectés par des phénomènes post-dépositionnels, ce qui permet une bonne préservation du matériel (fig. 8). De plus, malgré la rétention des spores et du pollen dans le réseau karstique, la diversité taxonomique ne semble pas affectée et paraît refléter la diversité botanique de l'environnement (McGarry \& Caseldine, 2004). Le tableau 2 présente l'inventaire de cette diversité pour l'ensemble des spéléothèmes étudiés, ainsi que le nombre minimal et maximal de taxons polliniques par spectre. Le filtre opéré par le réseau karstique génère une rétention quantitative, mais pas sélective ni différentielle. Le système, clos après la cristallisation de la calcite, permet de s'affranchir des phénomènes de contamination et de conservation différentielle. Dès lors, la validation des spectres polliniques issus de spéléothèmes est possible et permet de reconstituer la végétation.

\section{Baume Bonne}
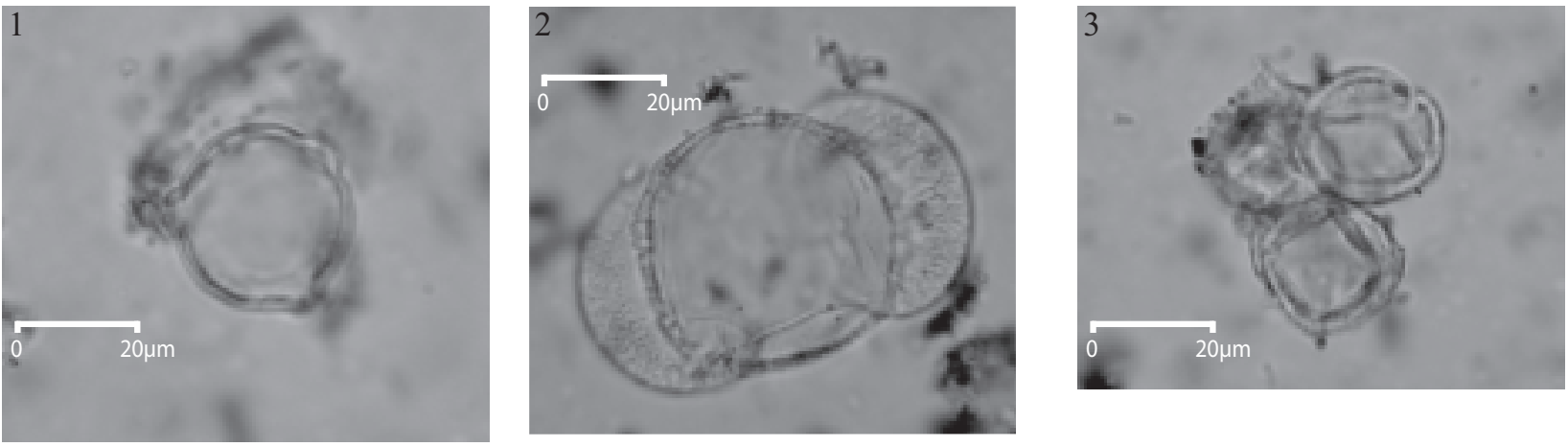

\section{Basura}
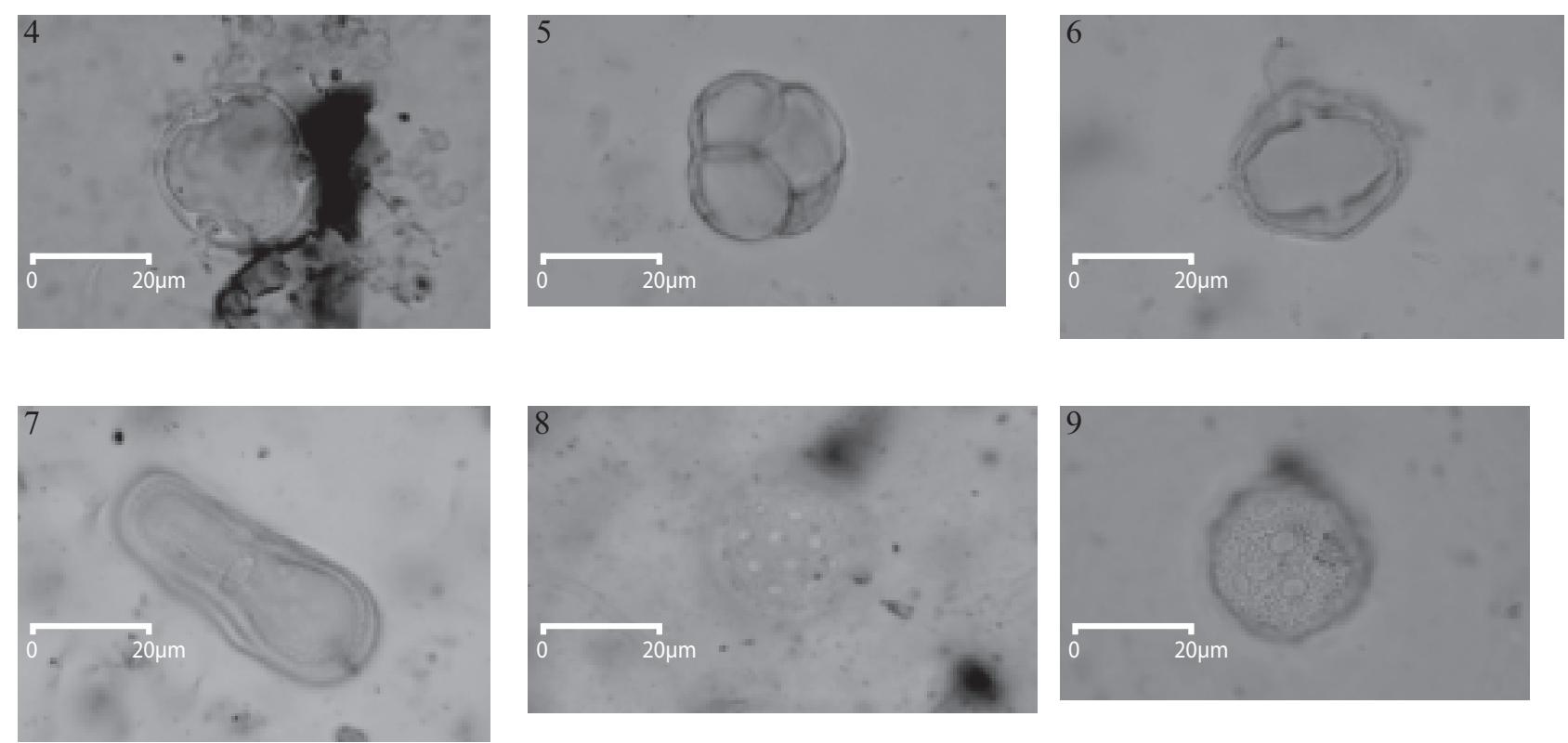

Fig. 8 : Exemples de grains de pollen bien conservés extraits des spéléothèmes. 1. Carpinus t. orientalis, 2. Pinus, 3. Quercus t. ilex/coccifera, 4. Tilia, 5. Ericaceae, 6. Hedera, 7. Apiaceae, 8. Chenopodiaceae, 9. Caryophyllaceae.

Fig. 8: Well-preserved pollen grains extracted from speleothems. 1. Carpinus t. orientalis, 2. Pinus, 3. Quercus t. ilex/coccifera, 4. Tilia, 5. Ericaceae, 6. Hedera, 7. Apiaceae, 8. Chenopodiaceae, 9. Caryophyllaceae. 


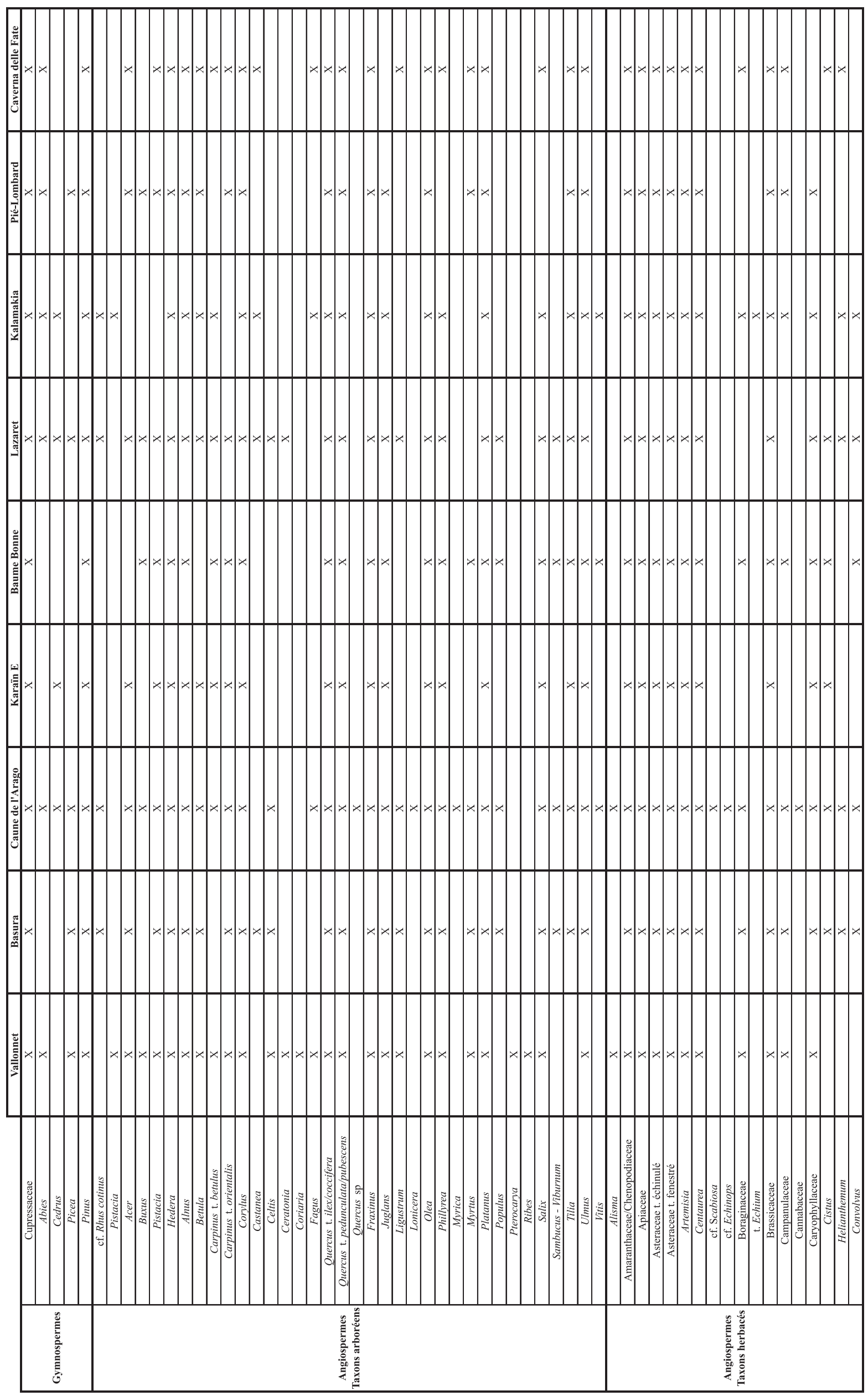




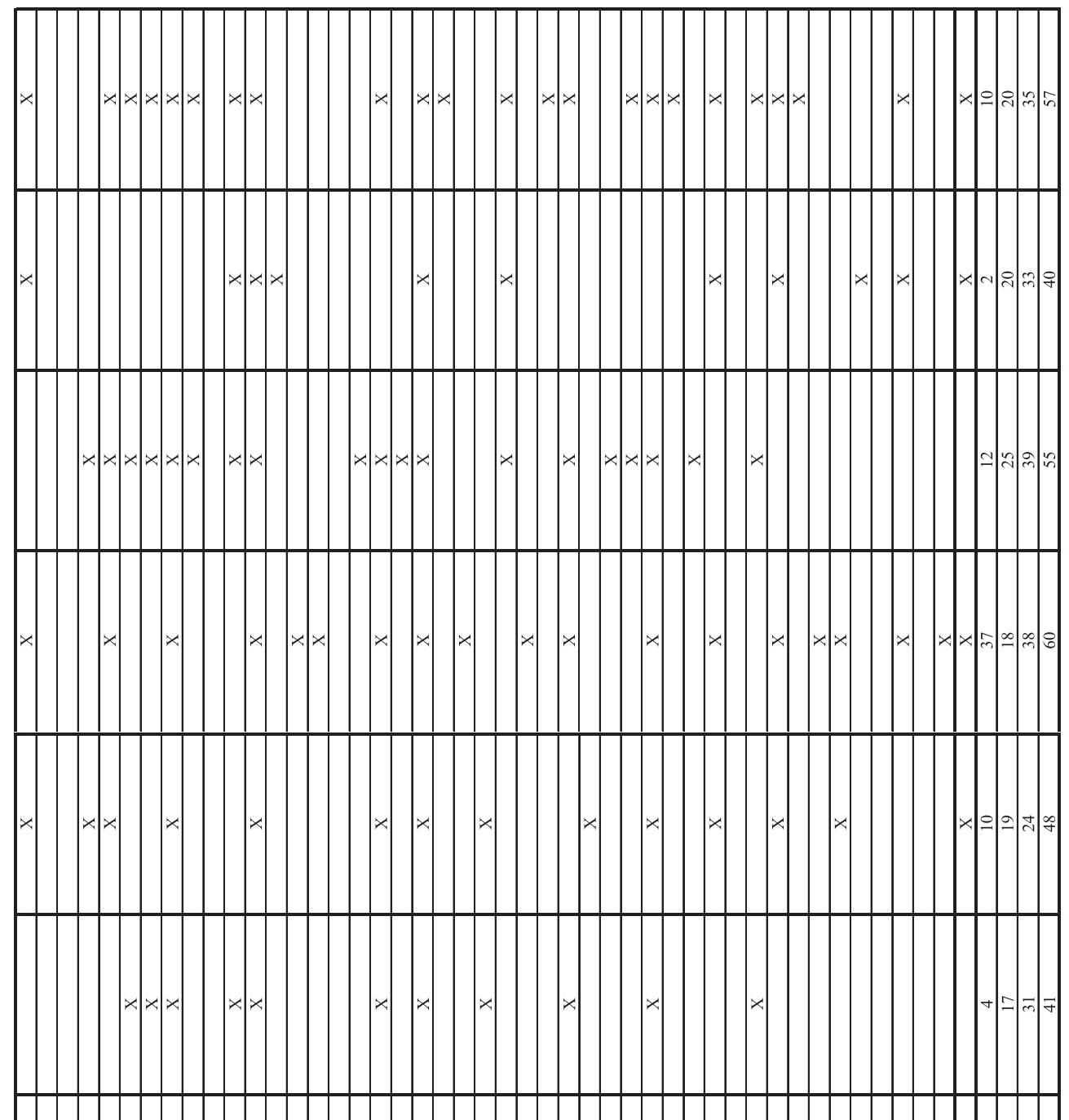

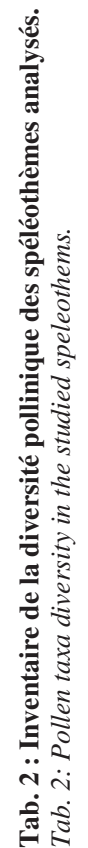

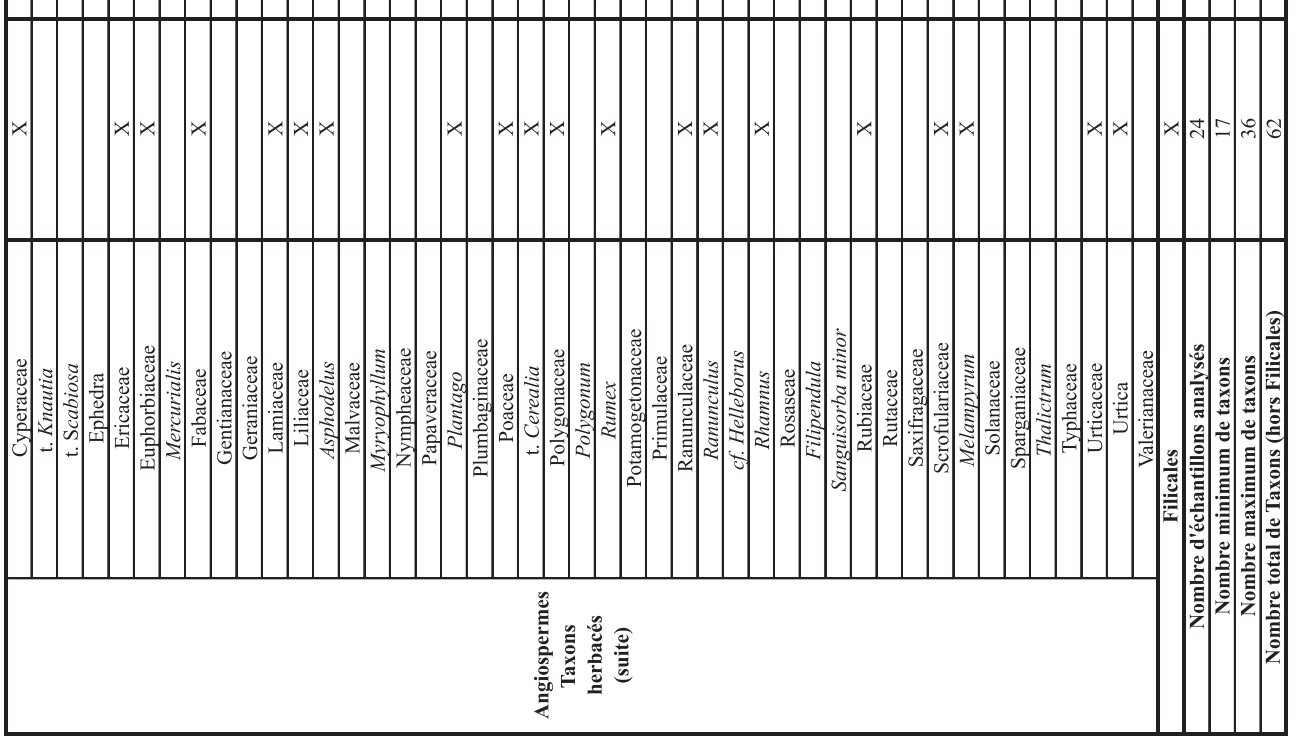




\section{3 - RELATION SPECTRES POLLINIQUES/ VÉGÉTATION}

Les obstacles à la reconstitution de la végétation à partir des spectres polliniques de spéléothèmes proviennent de l'estimation de la part des végétations locale et régionale. Il est également nécessaire de considérer l'origine, éolienne ou hydrique, du matériel pollinique (Genty et al., 2001) et la position du spéléothème dans la cavité (Van Campo \& Leroi-Gourhan, 1956 ; Smithson, 1982; Burney \& Burney, 1993; Coles \& Gilberston, 1994).

\subsection{1 - Distinction entre végétation locale et régionale}

Au Lazaret, l'ensemble stratigraphique E se compose de spéléothèmes variés. Le deuxième échantillonnage du dôme stalagmitique (fig. 4) permet d'établir un diagramme pollinique (fig. 9). Les spectres obtenus enregistrent la représentation importante des éléments de la forêt méditerranéenne et caducifoliée. Une telle représentation des taxons méditerranéens ne se rencontre pas dans les très longues séquences continentales (Wijmstra, 1969 ; Wijmstra \& Smit, 1979 ; Follieri et al., 1988 ; Pons \& Reille, 1988 ; Tzedakis, 1993, 1999, Tzedakis et al., 2006 ; Allen et al., 2000). Le développement de taxons thermophiles tels que Olea, autour de la grotte, peut s'expliquer par la proximité du littoral. La formation du spéléothème par ruissellement favorise l'enregistrement de la végétation méditerranéenne locale. Il est donc difficile de rapprocher la reconstitution de l'environnement végétal des épisodes climatiques globaux aride/humide du MIS 5. Les données antérieures pour cet ensemble E (Gauthier, 1992) diffèrent de ces nouveaux résultats. Les échantillons, prélevés dans le même ensemble stratigraphique mais dans des zones éloignées de la grotte, peuvent induire des décalages stratigraphiques et chronologiques à l'origine des différences dans les deux enregistrements polliniques.

L'analyse pollinique d'un spéléothème intercalé dans les niveaux moustériens de Karaïn E, permet de reconstruire un paléoenvironnement original. Cette stalagmite a enregistré l'image pollinique d'une végétation dominée par les arbres, en particulier par Fraxinus, avec une proportion relative moyenne de $20 \%$ (fig. 10). Ce signal se compose de marqueurs de la chênaie mésophile, de formations méditerranéennes, ainsi que d'une bonne représentation des herbacées. Néanmoins, l'ensemble de végétation dominant reste la ripisylve (Messager, 2002). La mise en évidence de cet environnement hygrophile s'accorde avec de nombreux autres résultats. La faune retrouvée à Karaïn $\mathrm{E}$ comporte Hippopotamus (Yalçinkaya, 1988 ; Bayon,

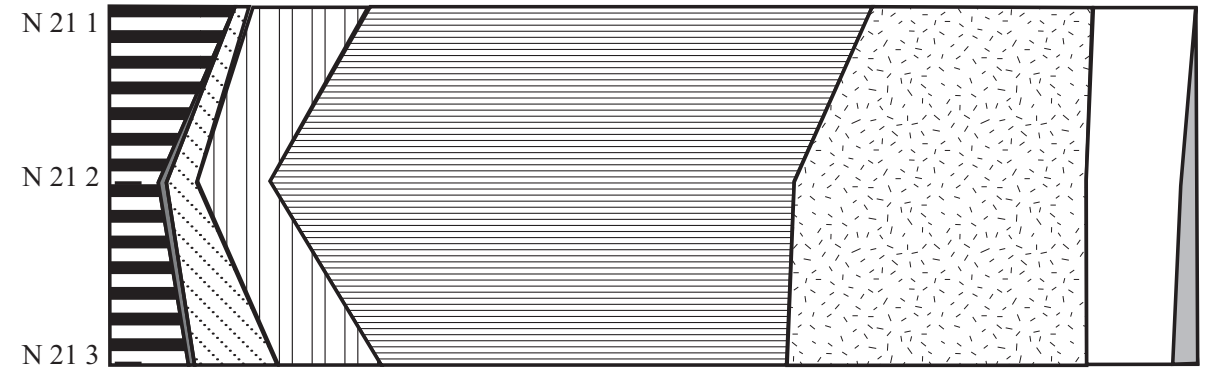

$*$

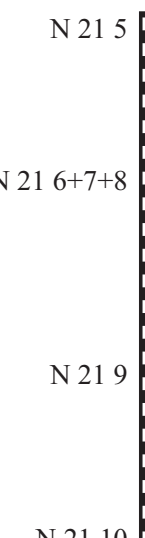

N 2110

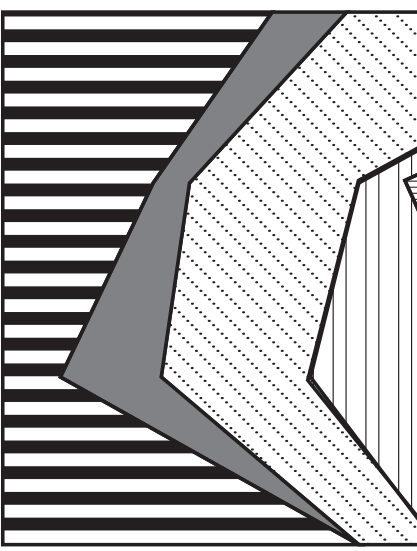

$0 \%$

Plancher stalagmitique

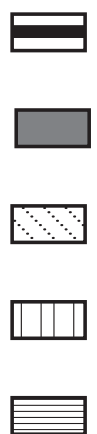

Gymnospermes

Betula

Forêt tempérée

caducifoliée

Taxons de ripisylve

Forêt sclérophylle méditerranéenne

Taxons steppiques et/ou xérophiles

Taxons ubiquistes

Filicales

Fig. 9 : Diagramme pollinique synthétique du dôme stalagmitique du Lazaret.

Fig. 9: Synthetic pollen diagram of a stalagmitic dome from Lazaret. 


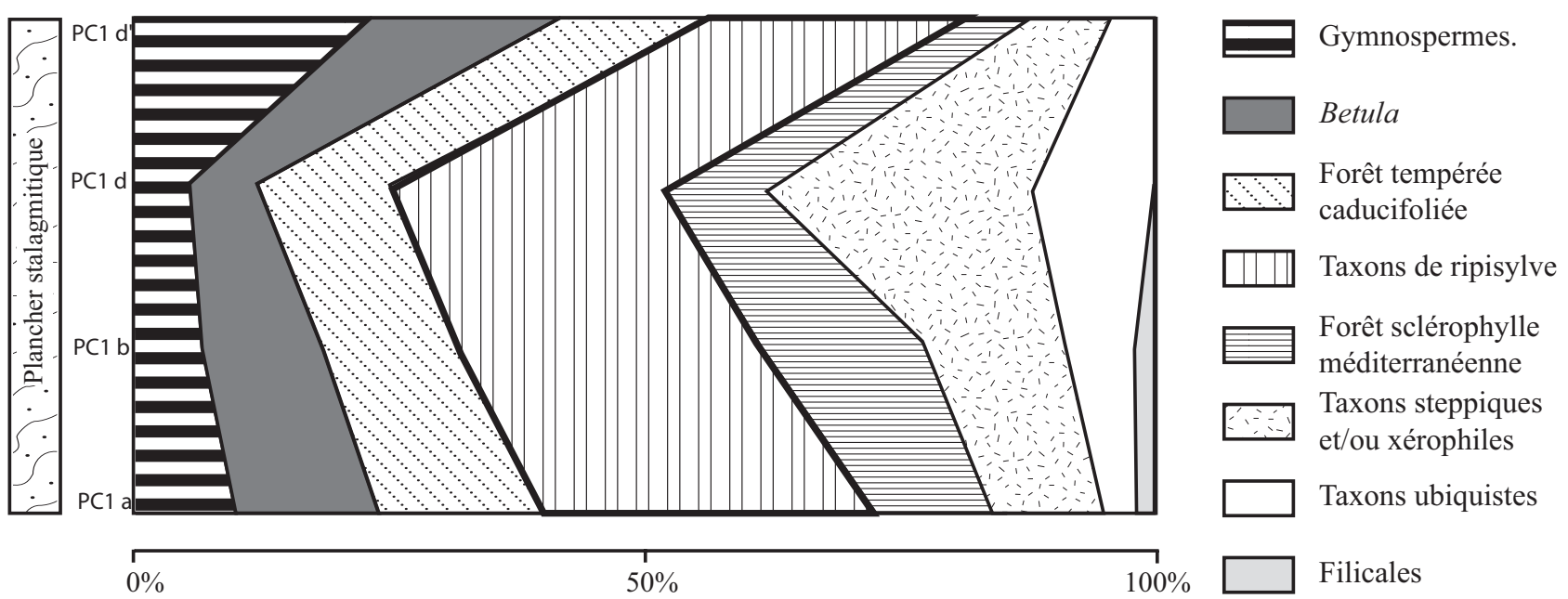

Fig. 10 : Diagramme pollinique synthétique de la stalagmite $\mathrm{C} 1$ de Karaïn $\mathrm{E}$.

Fig. 10: Synthetic pollen diagram of stalagmite C1 from Karaïn E.

1998), ainsi qu'une avifaune et une herpétofaune caractéristiques de milieu humide (Bailon \& Roger, inédit). La stalagmite étudiée enregistre une sur-représentation pollinique d'un écosystème local qu'il n'est pas envisageable d'extrapoler à l'échelle régionale. Ces apports polliniques, témoin d'une zone humide, proviennent plus vraisemblablement de la plaine proche et en contrebas du site que du plateau. Bien que cette flore pollinique témoigne d'un environnement très localisé, une origine éolienne est donc à retenir. Les apports éoliens peuvent donc refléter une part importante des environnements locaux.

\subsection{2 - Végétation stationnelle et interprétation de l'assemblage pollinique}

L'entrée des grottes peut favoriser l'installation d'une végétation adaptée à des conditions stationnelles particulières (paroi, degré d'ensoleillement, humidité, orientation, etc.). Ces plantes sciaphiles et saxicoles peuvent s'ajouter aux autres composantes de la végétation dans les spectres polliniques.

A Caverna delle Fate, un pic de Hedera (40\%), enregistré dans un seul échantillon, suggère l'abondance de cette plante près de l'entrée du site (Karatsori, 2003, 2005). Cette hypothèse se trouve renforcée par la présence d'amas polliniques de ce taxon dans l'échantillon. L'ensemble de l'étude pollinique indique un paysage semi-fermé à fermé, établi sous des conditions climatiques tempérées à caractère méditerranéen (fig. 11). Au sein de la zone pollinique Fat.3, l'enregistrement d'une brève période d'ouverture du paysage traduit une augmentation de l'aridité. De toute évidence, l'abondance de Hedera dans cet enregistrement ne perturbe pas les interprétations paléoenvironnementales et paléoclimatiques.

\subsection{3 - Position du spéléothème dans la cavité}

Selon la configuration du site ou la distance entre le spéléothème et l'entrée de la cavité, l'importance des apports éoliens est variable (Van Campo \& LeroiGourhan, 1956 ; Smithson, 1982 ; Burney \& Burney, 1993 ; Coles \& Gilberston, 1994). La végétation régionale peut alors être mieux représentée aux dépens des ensembles de végétation locale dans les spectres polliniques.

Dans l'abri de Pié-Lombard, les prélèvements de spéléothèmes ayant livré des spectres polliniques sont situés près de l'entrée actuelle du site (Renault-Miskovsky \& Texier, 1980). Ils concernent d'une part un échantillon de l'enveloppe externe d'un gros fragment de stalactite détaché du plafond de la cavité et d'autre part un échantillon de plancher stalagmitique constitué de 3 niveaux difficiles à séparer, la calcite épousant les reliefs du sol rocheux. Ces niveaux carbonatés se sont formés après l'ouverture de la grotte et les apports polliniques sont certainement d'origine éolienne. Le contenu sporo-pollinique des deux échantillons présente les mêmes caractéristiques (fig. 12). AP est voisin de $50 \%$ et le couvert arboré est dominé par les pollens anémogames comme Pinus, accompagné par quelques taxons montagnards. Les essences caducifoliées tempérées sont nombreuses en genres mais peu représentées par leur nombre de grains. Cependant la variété des taxons thermophiles ou franchement méditerranéens suggère que les concrétions se sont constituées sous un climat à cachet méditerranéen, mais plus humide que l'actuel. Cette image pollinique traduit un environnement contemporain d'un climat interglaciaire. Cette reconstitution s'oppose très nettement au paysage découvert steppique évoqué par l'étude pollinique du remplissage archéologique moustérien et mis en place sous un climat froid et sec au début du MIS 4, daté aux environs de 70000 ans par thermoluminescence (Valladas et al., 1987, 1988). Cette période interglaciaire se placerait donc au MIS 5.

A Kalamakia, le pilier stalagmitique analysé est placé à l'entrée de la grotte dont le porche mesure près de $10 \mathrm{~m}$ de large et de haut. Deux zones polliniques se 


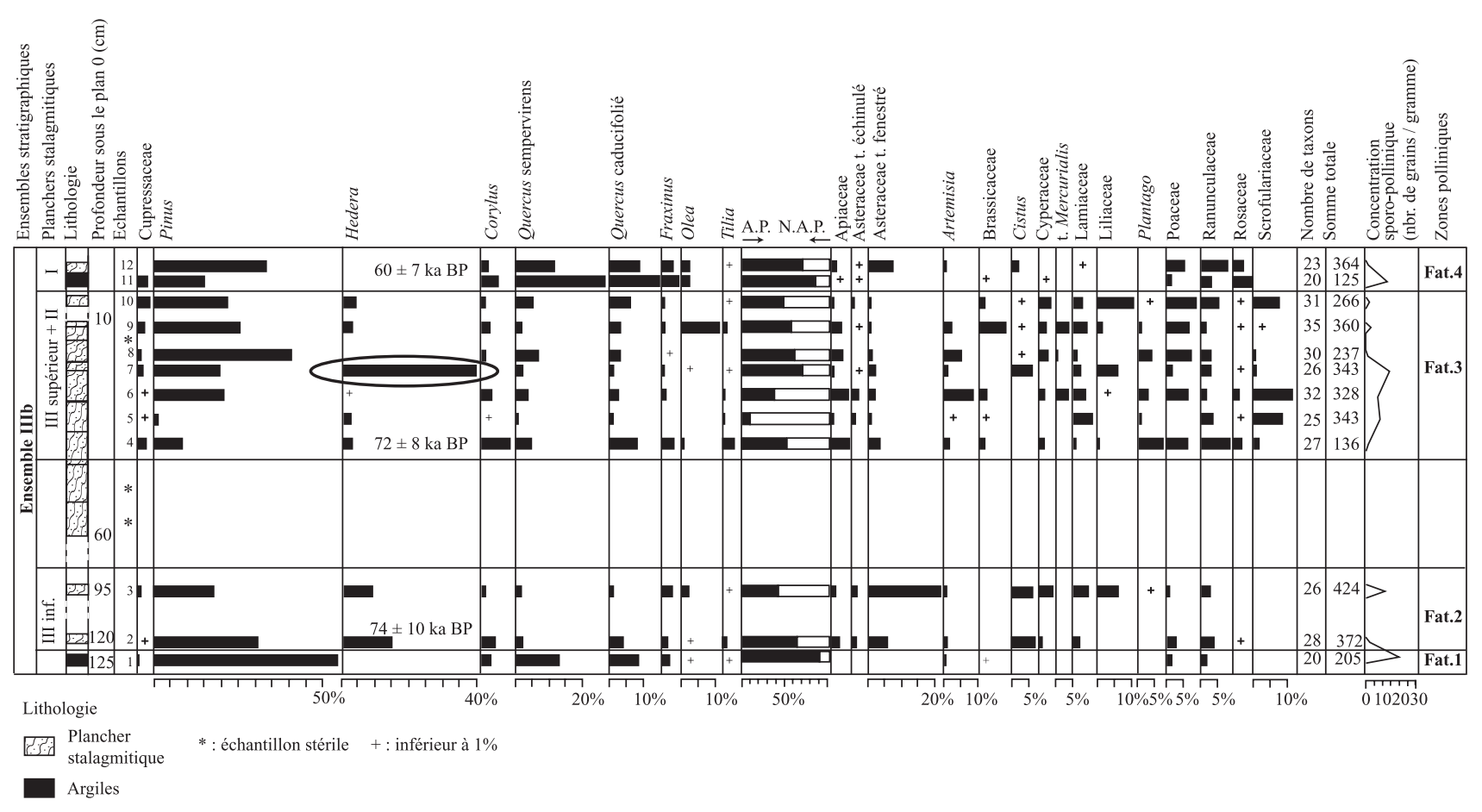

Fig. 11 : Diagramme pollinique détaillé des taxons les plus significatifs de Caverna delle Fate.

Fig. 11: Detailed pollen diagram of the most significant taxa from Caverna delle Fate.

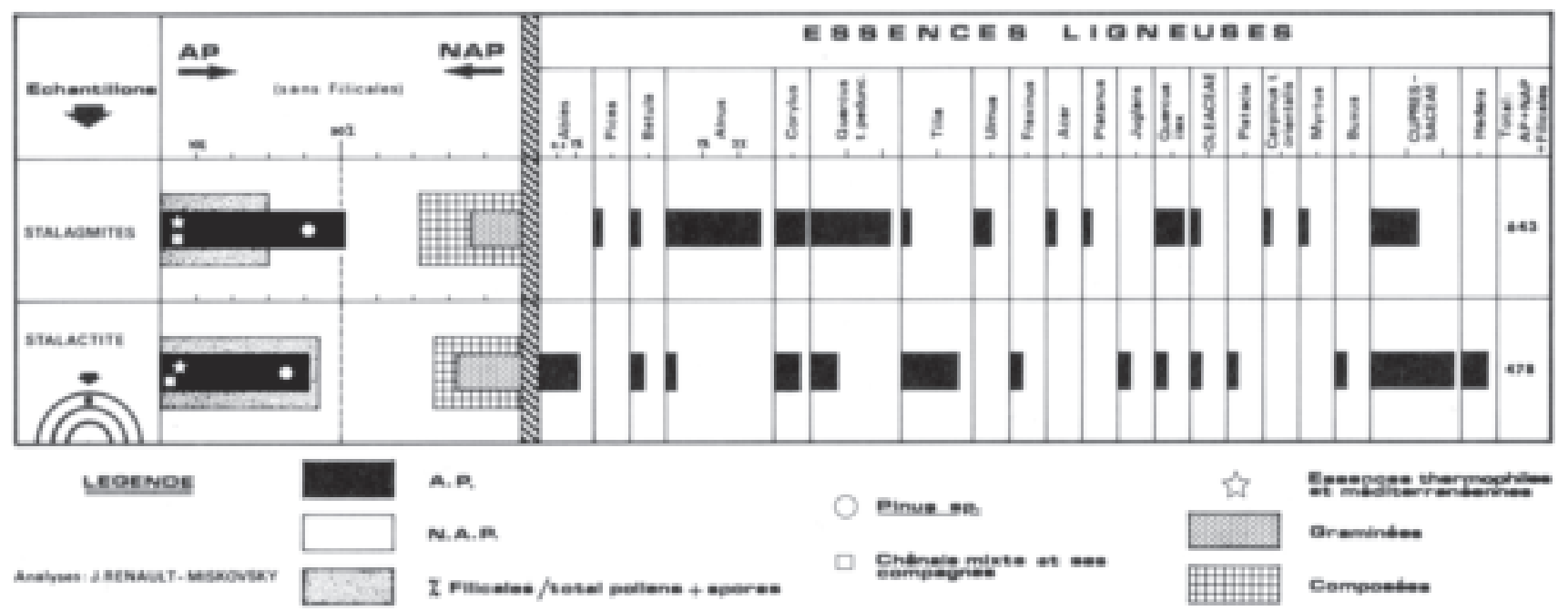

Fig. 12 : Diagramme pollinique détaillé des taxons les plus significatifs de Pié-Lombard.

Fig. 12: Detailed pollen diagram of the most significant taxa from Pié-Lombard.

distinguent (fig. 13). Dans la première, une forêt caducifoliée est enregistrée avec les arbres mésophiles et hygrophiles bien diversifiés. Pinus n'est jamais surreprésenté, à l'exception d'une hausse brutale et ponctuelle dans un échantillon. Les Poaceae sont les seules herbacées bien représentées. Dans la deuxième zone, les taxons herbacés et arborés formant un assemblage pré-forestier méditerranéen à caractère steppique progressent aux dépens des arbres caducifoliés. Parmi ceux-ci, seuls Quercus et Fraxinus subsistent. Cette modification apparente du couvert végétal pendant la formation du spéléothème peut traduire un épisode d'aridité en terme climatique. Il peut aussi s'expliquer par un changement dans la proportion des apports polliniques éoliens et hydriques au sein des spectres analysés. Les spectres polliniques enregistrent tant la part de la végétation locale pouvant être apportée par l'eau via le réseau karstique, que celle de la végétation régionale plutôt liée à des apports éoliens. L'analyse présente alors une image composite superposant les différents ensembles de végétations caducifoliée et méditerranéenne. 


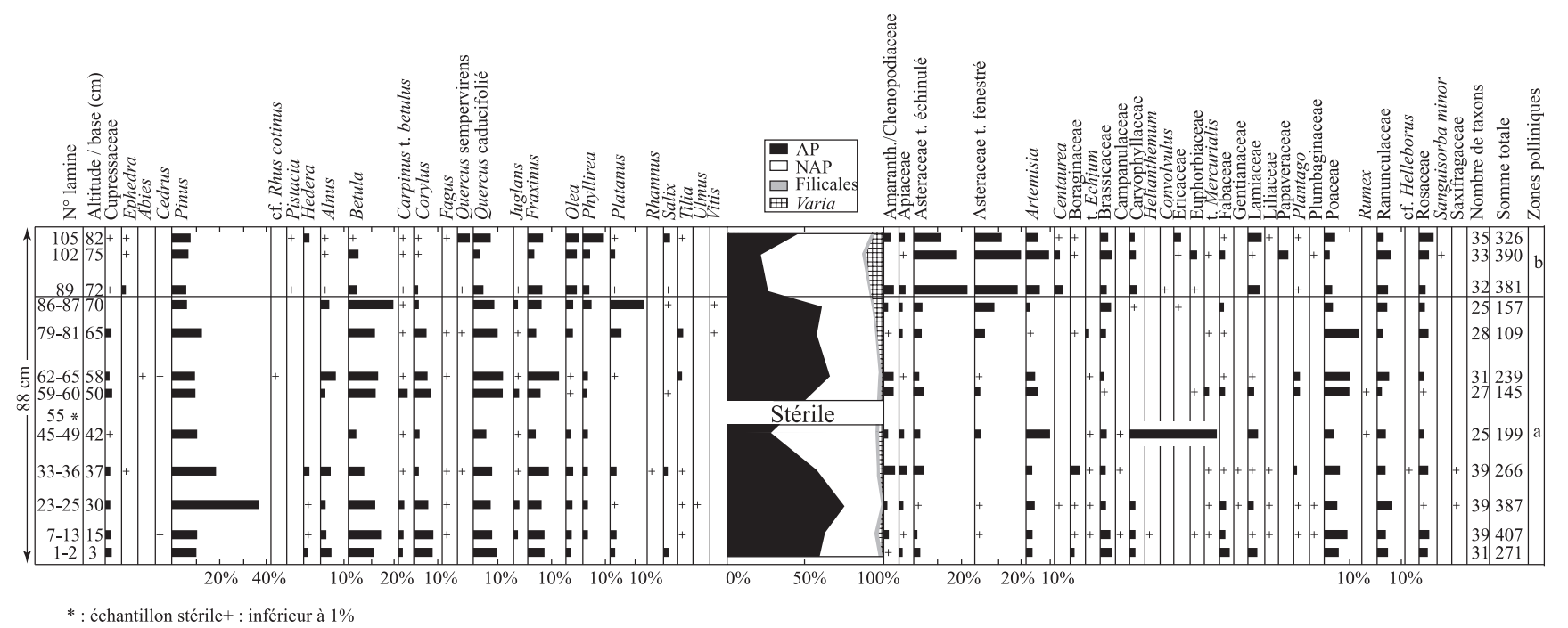

Fig. 13 : Diagramme pollinique détaillé du pilier stalagmitique de Kalamakia.

Fig. 13: Detailed pollen diagram of the large stalagmite from Kalamakia.

\section{4 - RELATION SPÉLÉOTHÈME/PALÉOENVIRON- NEMENT/OCCUPATION PRÉHISTORIQUE}

\subsection{1 - Intégration de la palynologie aux études multidisciplinaires}

Les spéléothèmes supportent le développement de nombreuses études pluridisciplinaires (analyses isotopiques, fluorescence, études radiochronologiques, chronostratigraphiques, paléoenvironnementales, paléoclimatiques, etc.). Si l'aspect climatique est perçu de manière globale par les analyses isotopiques (Lauritzen \& Lundberg, 1999), la palynologie appliquée aux spéléothèmes fournit une information environnementale de dimensions spatiales beaucoup plus réduites. Les données polliniques, couplée aux datations radiométriques des spéléothèmes analysés, permettent de reconstituer l'environnement au moment de sa formation. Deux exemples, parmi les gisements présentés ici, sont détaillés pour montrer la complémentarité des résultats issus de ces disciplines avec les données de la palynologie.

Les spéléothèmes de l'ensemble stratigraphique $\mathrm{E}$ de la grotte du Lazaret ont fait l'objet d'analyses isotopiques et géochimiques (Rousseau et al., 2005). Les analyses isotopiques de l'oxygène et du carbone permettent d'identifier les sous-stades du MIS 5, en accord avec les datations TIMS. Ces courbes montrent des variations synchrones de celles à caractère global enregistrées dans les carottes marines. Si les spectres polliniques des analyses antérieures (Gauthier, 1992) et de la nouvelle étude (fig. 9) soulignent des divergences, sans doute liées aux localisations des échantillons, ils révèlent la présence de Pinus et Betula. Or, l'étude des stérols montre la présence des terpénoïdes, caractéristique de Pinus sp., et de la bétuline, caractéristique de Betula. Ces deux taxons polliniques se développaient probablement aux environs immédiats de la grotte.
Plusieurs carottes de près de deux mètres de long ont été prélevées récemment à la Basura. Sur des échantillons ponctuels du plancher de fermeture, datés respectivement de $12 \mathrm{ka}$ et $110 \mathrm{ka}$ B.P. (Yokoyama et al., 1985), des œufs d'Helminthes ont été déterminés sur les lames polliniques (Lartigot \& Croizet, 2005). L'absence de traitement chimique à la potasse des échantillons expliquerait cette présence. Il s'agit notamment d'œufs de Opisthorchis/Clonorchis sp. qui peuvent avoir pour hôte des mangeurs de poissons d'eau douce. Des œufs de Trichurideae, qui peuvent avoir pour hôte les Suideae ou l'Homme, ont également été rencontrés.

\subsection{2 - Jalons paléoenvironnementaux et chronologiques}

Les résultats palynologiques peuvent concerner des spéléothèmes encadrant des ensembles archéologiques ou formant des unités stratigraphiques à part entière. Les informations délivrées permettent de reconstituer ponctuellement l'évolution des paysages quaternaires et constituent ainsi des jalons paléoenvironnementaux et paléoclimatiques.

Le plancher stalagmitique de base et le plancher de fermeture qui scelle le remplissage de la grotte du Vallonnet illustrent ce point. Selon Renault-Miskovsky \& Girard (1976, 1978, 1988), l'étude palynologique de la totalité du remplissage du Vallonnet révèle cinq ensembles en terme de végétation et de climat (fig. 14). Le premier et le quatrième concernent les spéléothèmes de base et de fermeture, encadrant le remplissage sédimentaire contemporain de l'occupation humaine. Le plancher stalagmitique de base révèle l'existence d'une forêt de feuillus à Platanus et à Pterocarya, accompagnés de Pinus et de taxons méditerranéens. Ce couvert forestier a pu s'établir sous un climat chaud et plus humide que le climat méditerranéen actuel, à valeur d'interglaciaire, au Pléistocène inférieur il y a environ 1,4 Ma. Au niveau de la stalagmite de fermeture, la 


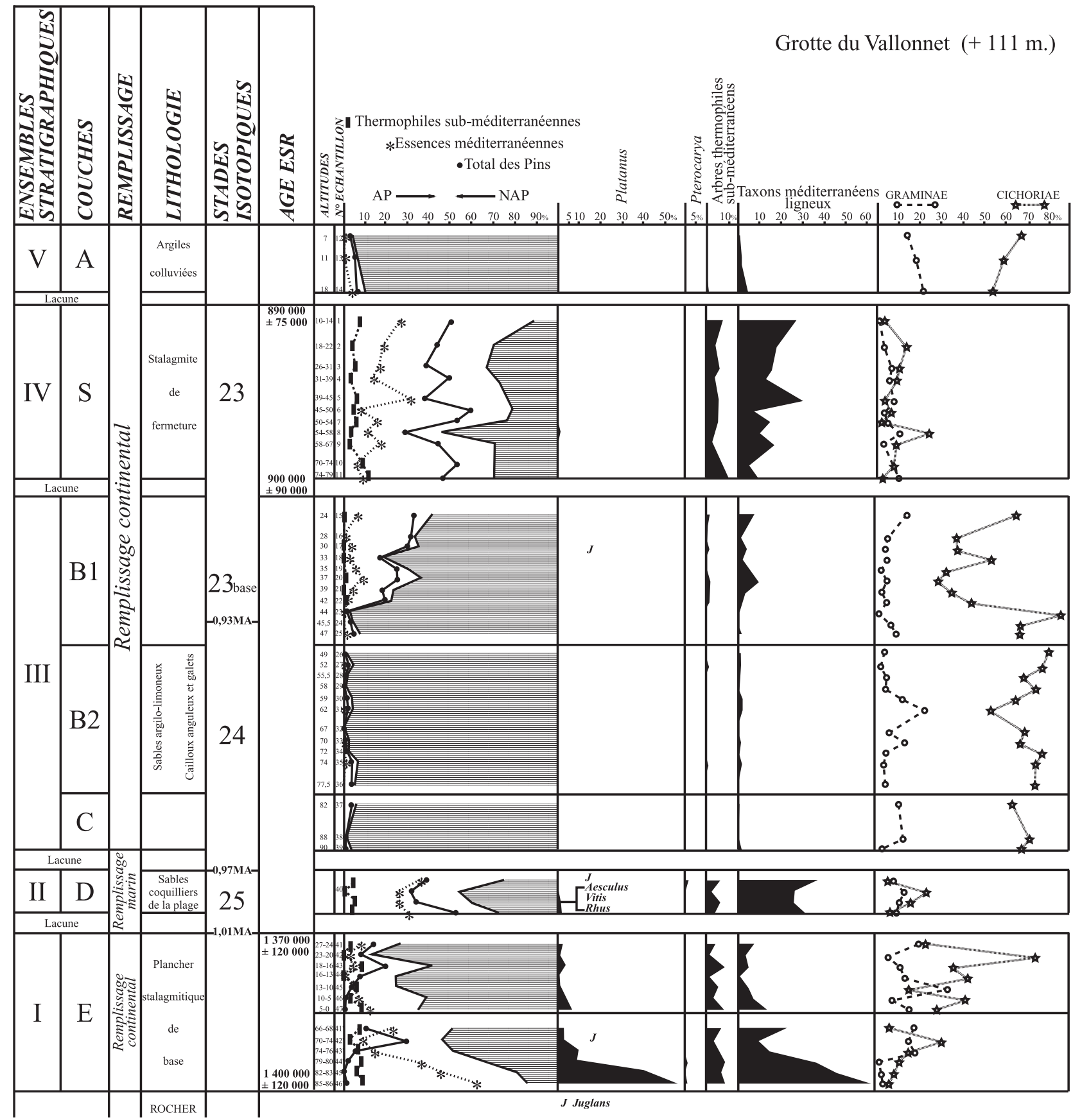

Fig. 14 : Diagramme pollinique de la grotte du Vallonnet.

Fig. 14: Pollen diagram from Vallonnet.

composition forestière est riche, dominée par la pinède, suivie par les essences méditerranéennes et les caducifoliés, exceptés Platanus et Pterocarya. Les conditions climatiques sont vraisemblablement interglaciaires. Cet épisode fait suite à la période froide et sèche durant laquelle s'étendait une steppe contemporaine de la fréquentation du site par des Hominidés. Les quelques essences reliques thermophiles qui persistaient encore dans le plancher de base ont totalement disparu dans la stalagmite de fermeture datée de 0,9 Ma. Ces spéléothèmes constituent donc deux jalons thermophiles pour des épisodes climatiques de type interglaciaire du Pléistocène inférieur, bien datés et en stratigraphie, contrastant fortement avec le paysage largement découvert des niveaux archéologiques intercalés entre ces deux ensembles.

Le remplissage de la Caune de l'Arago est subdivisé en plusieurs ensembles stratigraphiques, dont certains renferment des spéléothèmes (Lumley de et al., 1984). Le premier ensemble consiste en un plancher stalagmitique reposant directement sur l'encaissant calcaire, daté par ESR de $700 \mathrm{ka}$ (Yokoyama et al., 1982). Son analyse palynologique montre un couvert arboré bas et ouvert, laissant envisager un climat à tendance méditerranéenne, assez chaud et relativement sec (Renault-Miskovsky, 1995). Reposant directement sur cette formation, une épaisse séquence détritique englobe plusieurs sols d'occupation riches en matériel 
archéologique. Sur ces sédiments repose le second ensemble de spéléothèmes constitué de coulées stalagmitiques. Des datations récentes ont établi sa mise en place entre 350 et 104 ka B.P. (Falguères et al., 2004). Les analyses polliniques (fig. 15) révèlent qu'une formation méso-méditerranéenne proche de l'actuelle régnait aux alentours du site à cette époque, en accord avec les précédentes études (Rahardjo, 1984 ; Gauthier, 1984, 1992). Cependant, quelques apports plus lointains se superposent à cette image, comme la ripisylve des bords du Verdouble et un écho de la végétation régionale. Enfin, le dernier ensemble de spéléothèmes clos le remplissage. Son âge est estimé entre 100 et $34 \mathrm{ka}$ (Shen, 1985). Les analyses palynologiques montrent la présence d'une strate herbacée abondante, ponctuée de ligneux méditerranéens, témoignant du développement de la garrigue aux alentours du site, sous un climat chaud et sec (RenaultMiskovsky, 1995).

Dans l'abri de Pié-Lombard, le contenu sporo-pollinique des spéléothèmes affleurants dans le remplissage archéologique constitue un jalon paléoenvironnemental. Par contre, il ne forme pas un jalon chronologique en l'absence de datation sur les spéléothèmes. La période tempérée à cachet méditerranéen enregistrée tranche avec le paysage découvert steppique établi sous un climat froid et sec pendant le MIS 4, lors de l'occupation humaine moustérienne (Renault-Miskovsky \& Texier, 1980).

\subsection{3 - Impact de l'occupation préhistorique sur le signal pollinique}

Quand les spéléothèmes analysés sont directement corrélés avec les occupations humaines d'un gisement préhistorique, le flux pollinique peut enregistrer des perturbations directement liées aux occupants de la grotte, que ce soit les Hommes ou les animaux, évoluant dans l'écosystème proche du site (Martin \& Sharrock, 1964 ; Leroi-Gourhan, 1966, 1981 ; Davis \& Anderson, 1987 ; Bui-Thi-Mai \& Girard, 1988, 2000 ; Diot, 1991 ; Navarro et al., 2000 ; Carrión et al., 2001, 2005). Le passage d'animaux ou l'occupation d'un site

Caune de l'Arago, Tautavel, France Altitude: $+210 \mathrm{~m}$

Ensemble stratigraphique IV, Pilier stalagmitique Nord

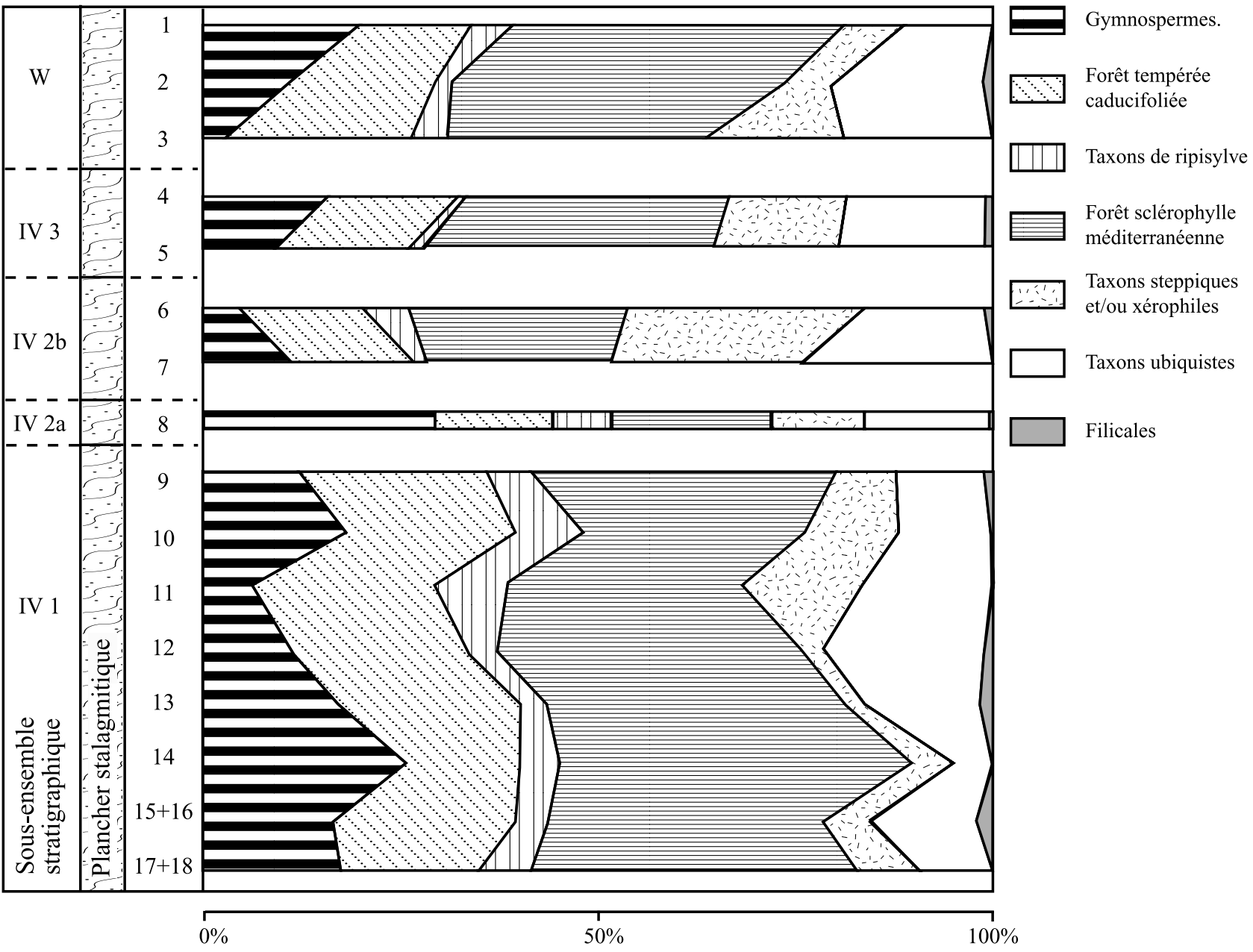

Fig. 15 : Diagramme pollinique synthétique des coulées stalagmitiques formant le deuxième ensemble de spéléothèmes de l'Arago.

Fig. 15: Synthetic pollen diagram of the stalagmites of the second speleothem unit from Arago. 


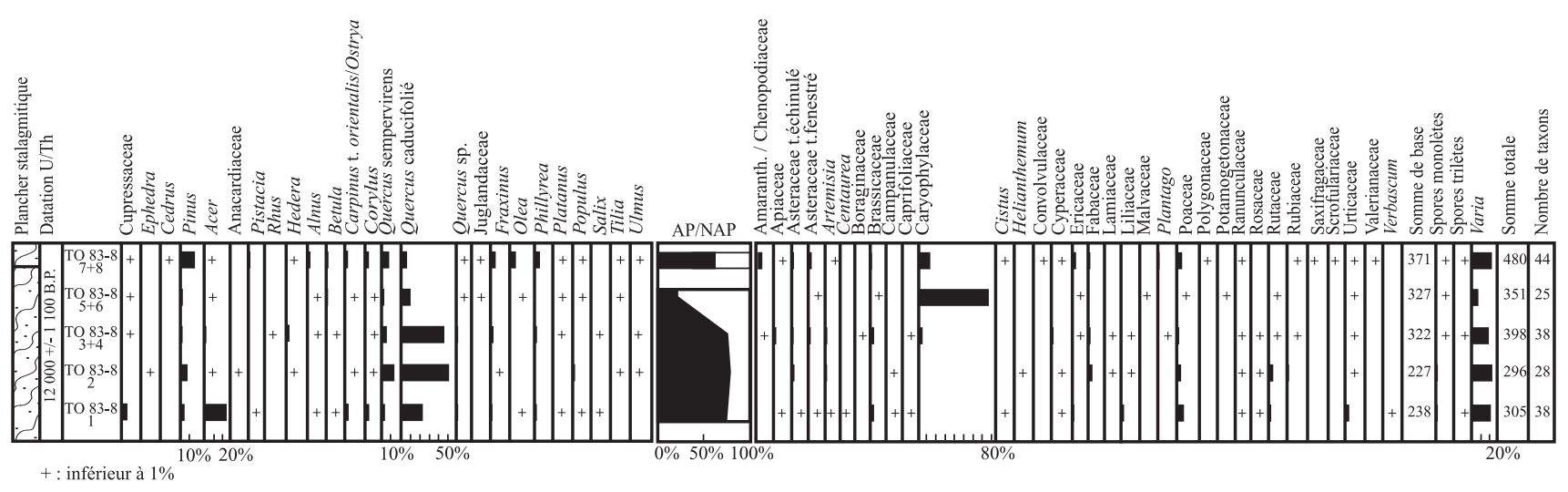

Fig. 16 : Diagramme pollinique détaillé du plancher stalagmitique de fermeture de la Basura (colonne TO 83-8). Fig. 16 : Detailed pollen diagram of the upper stalagmitic floor from Basura (sampling TO 83-8).

par des Hommes préhistoriques peuvent être des vecteurs accidentels de pollen et la sur-représentation d'un taxon est de ce fait perceptible dans les analyses polliniques de spéléothèmes.

A la Basura, les analyses palynologiques du sommet du plancher de fermeture TO 83-8 livrent cinq spectres fournissant un nombre de pollen suffisant pour reconstituer le paléoenvironnement végétal (fig. 16). Dans deux niveaux, le pourcentage de Caryophyllaceae est très élevé, atteignant jusqu'à 76,5\%. Des prélèvements latéraux dans la colonne TO 83-19 du même plancher (fig. 6) ne montrent pas de telles fréquences, les Caryophyllaceae étant toujours inférieures à $5 \%$. Cette divergence traduit une introduction très ponctuelle dans le spéléothème.

A Kalamakia, un pic de Caryophyllaceae $(37,5 \%)$ se rencontre également dans un échantillon, alors que ce taxon est absent dans les spectres précédents et suivants (fig. 13). Cette soudaine augmentation, qui entraîne la variation brutale du rapport AP/NAP, est donc sans signification paléoécologique.

\subsection{4 - Spéléothèmes et environnement de l'Homme fossile}

Les spéléothèmes, quand ils sont corrélés avec les niveaux archéologiques, permettent d'approcher le plus fidèlement possible l'environnement végétal de l'Homme préhistorique. Des neuf sites présentés pour cette étude, seuls les sites de Karaïn E et Kalamakia montrent cette contemporanéité entre les sols archéologiques et la formation de la calcite. Dans les deux cas, l'analyse pollinique des spéléothèmes a pour but de préciser les conditions environnementales synchrones de l'occupation du site par des Hominidés, afin de mieux comprendre les comportements de subsistance de ces populations, lors du dernier cycle climatique.

Le spéléothème étudié à Karaïn $\mathbf{E}$ (fig. 10) bénéficie d'un calage chronologique relativement précis. Les lamines de la base et du sommet du spéléothème sont datées par la méthode U/Th de $124+25 /-19$ ka et 120 +32/-21 ka, situant la stalagmite à la base du MIS 5 (Farkh, 2004). Les caractéristiques climatiques du MIS 5e ont sans doute permis l'établissement d'une végétation méditerranéenne et caducifoliée dans ce secteur. Par ailleurs, l'accroissement des précipitations au cours de cet épisode semble compatible avec le développement de points d'eau expliquant la présence de ripisylves. Ce spéléothème situé en relation stratigraphique directe avec les niveaux archéologiques moustériens apporte donc une information chronologique et une image assez précise de l'environnement immédiat de la grotte lors de son occupation.

En Grèce, l'Homme de Néandertal de Kalamakia évoluait dans un environnement forestier marqué progressivement par un épisode d'aridité voyant le recul des arbres au profit du couvert herbacé (fig. 13). Ces résultats s'accordent avec les données paléontologiques (Psathi, comm. pers.). La liste faunique montre l'abondance de Dama sp. et Capra sp. Ces taxons évoluent dans un environnement semi-ouvert comme le milieu pré-forestier méditerranéen à caractère steppique. La présence de Sus scrofa, Cervus elaphus et Capreolus capreolus s'accorde avec l'enregistrement pollinique des éléments arborés caducifoliés et hygrophiles. Ainsi, les études palynologique et faunistique menées conjointement soulignent les relations Homme/milieu au Paléolithique moyen, avec une spécialisation opportuniste des techniques de chasse face au gibier disponible dans l'environnement.

\section{5 - CONCLUSION}

La démarche méthodologique exposée démontre que les analyses palynologiques des spéléothèmes peuvent être des archives paléoenvironnementales fiables. Cependant, les caractéristiques propres à ce type de support conditionnent des processus taphonomiques particuliers susceptibles d'affecter le matériel sporo-pollinique. Les limites inhérentes à ces spécificités, notamment les faibles concentrations et la représentation locale de l'environnement, doivent alors être prises en considération. Les résultats, souvent ponctuels tant sur le plan spatial que chronologique, rendent délicates les comparaisons avec les séquences de référence et les hypothèses paléoclimatiques. Toutefois les qualités intrinsèques 
des spéléothèmes, comme l'absence de contamination et la diversité taxonomique, offrent la possibilité de reconstituer l'environnement. En contexte archéologique, ces spéléothèmes constituent une réelle opportunité de décrire le milieu végétal de l'Homme préhistorique. Les sites paléolithiques de cette étude se localisent sur le pourtour septentrional de la Méditerranée où les sédiments quaternaires propices à la conservation du pollen sont rares. Dans le cadre de l'étude multidisciplinaire d'un gisement, les spéléothèmes représentent alors un support d'analyse supplémentaire, source d'informations paléoécologiques essentielles. En relation stratigraphique avec des niveaux archéologiques, ils permettent même de reconstituer l'environnement aux abords d'un gisement préhistorique et d'appréhender ainsi les relations entre l'Homme et son milieu.

\section{RÉFÉRENCES BIBLIOGRAPHIQUES}

ALLEN J.R.M., WATTS W.A., \& HUNTLEY B., 2000 - Weichselian palynostratigraphy, palaeovegetation and palaeoenvironment ; the record from Lago Grande di Monticchio, southern Italy. Quaternary International, 73-74, 91-110.

AROBBA D., 1985 - Analisi palinologiche delle stalagmiti della grotta della Basura (Toirano, Liguria) : primi resulti. Revista di Studi Liguri, 51 (4), 354-359.

ATKINSON T.C., HARMON R.S., SMART P.L., \& WALTHAM A.C., 1978 - Paleoclimatic and geomorphic implications of $230 \mathrm{Th}$ / $234 \mathrm{U}$ on speleothems from Britain. Nature, 272, 24-28.

ATKINSON T.C., 1981 - The climate of Castleguard Cave, Canada. Proceedings of the Eighth International Speleological Congress, Bowling Green (Kentucky, USA), 322-324.

BAKER A., SMART P.L., EDWARDS R.L., \& RICHARDS D.A., 1993 - Annual growth bandings in cave stalagmite. Nature, $\mathbf{3 6 4}$ $518-520$

BAKER A., \& SMART P.L., 1995 - Recent flowstone growth rates : Field measurements in comparison to theoretical predictions. Chemical Geology, 122, 121-128.

BAKER A., BARNES W.L., \& SMART P.L., 1997 - Variations in the discharge and organic matter content of stalagmite drip waters in Lower Cave, Bristol. Hydrological Processes, 11, 533-537.

BASTIN B., 1971 - Recherches sur l'évolution du peuplement végétal en Belgique durant la glaciation du Würm. Acta Geographica Lovaniensa, 9, $136 \mathrm{p}$.

BASTIN B., 1978 - L'analyse pollinique des stalagmites : une nouvelle possibilité d'approche des fluctuations climatiques du Quaternaire. Annales de la Société Géologique de Belgique, 101, 13-19.

BAYON I., 1998 - La faune et les hommes du Paléolithique moyen de Karaïn (quelques notes préliminaires). In M. Otte (ed.), Préhistoire d'Anatolie, Genèse entre deux mondes. ERAUL, Liège, $\mathbf{8 5}$, $479-487$

BLANC J.J., 2005 - La croissance des spéléothèmes : un enregistrement climatique à haute résolution, analyses d'images, périodes passées et futures, essais d'interprétation. L'Anthropologie, 109 (2), 215-248.

BUI-THI M., \& GIRARD M., 1988 - Apports actuels et anciens de pollens dans la grotte de Foissac (Aveyron, France). Publication de l'Institut Français de Pondichéry, 25, 43-53.

BUI-THI-MAI, \& GIRARD M., 2000 - Les guanos de chauve-souris d'âge médiéval de la grande grotte d'Arcy-sur-Cure (Yonne) : analyse pollinique et implications paléobotaniques. Géologie alpine, 31, 83-95.

BURNEY D.A., \& BURNEY L., 1993 - Modern pollen deposition in cave sites : experimental results from New York State. New Phytologist, 124, 523-535.
BURNEY D.A., BROOK G.A., \& COWART J.B., 1994 - A Holocene pollen record from the Kalahari Desert of Botswana from a U-series dated spéléothème. The Holocene, 4, 225-232.

CARRASCO F., ANDREO B., BENAVENTE J., \& VADILLO I., 1995 - Chemistery of the water in the Nerja Cave System (Andallusia, Spain). Cave and Karst Science, 21, 27-32.

CARRION J.S., RIQUELME J.A., NAVARRO C., \& MUNUERA M., 2001 - Pollen in hyaena coprolites reflects late glacial landscape in southern Spain. Palaeogeography, Palaeoclimatology, Palaeoecology, 176, 193-205.

CARRION J.S., GIL G., RODRIGUEZ E., FUENTES N., GARCIA-ANTON M., \& ARRIBAS A., 2005 - Palynology of badger coprolites from central Spain. Palaeogeography, Palaeoclimatology, Palaeoecology, 226, 259-271.

CATTANI L., \& RENAULT-MISKOVSKY J., 1989 - La réponse des végétations aux variations climatiques quaternaires autour des sites archéologiques du sud de la France et du nord-est de l'Italie. Il Quaternario, 2 (2), 147-170.

CATTANI L., GARCIA-ANTON M., \& RENAULTMISKOVSKY J., 1994 - The Lower and Middle Pleistocene in the circum-Mediterranean region. Attempts and results of archeo-palynological correlations. American Association of Stratigraphic Palynologists Contributions Series, 29, 1-52.

CHIAPELLA V., 1955 - Grotta della Basura a Toirano (Savona). Quaternaria, 2, 282-284.

COLES G.M., GILBERSTON D.D., HUNT C.O., \& JENKINSON R.D.S., 1989 - Taphonomy and palynology of Cave Deposits. Cave Science, 16, 83-89.

COLES G.M., \& GILBERSTON D.D., 1994 - The airfall-pollen budget of archaeologically important sites : Creswell Crags, England. Journal of Archaeological Science, 21, 735-755.

DAVIS O.K., \& ANDERSON R.S., 1987 - Pollen in packrat (Neotoma) middens : pollen transport and the relationship of pollen to vegetation. Palynology, 11, 185-198.

DELCOURT A., MULLENDERS W., \& PIERART P., 1959 - La préparation des spores et des grains de pollen. Les naturaliste belges, 40, 92-120.

DELIBRIAS G., 1985 - Les dates ${ }^{14} \mathrm{C}$. Revue d'Etudes Ligures, 51 (4), 379-380.

DIJKSRA S.J., 1946 - Eine monographische Bearbeitung der Karbonischen Megasporen mit besonderer Berücksichtigung von SüdLimburg (Niederlande). Mededelingen van de Geologische Stichting, Série C, III, 1 (1), $101 \mathrm{p}$.

DIOT M.F., 1988 - Etudes palynologiques du remplissage de la grotte Vauffrey. In J.P. Rigaud (ed.), La grotte Vauffrey à Cénac et Saint-Julien (Dordogne, paléoenvironnements, chronologie et activités humaines. Mémoire de la Société Préhistorique Française, 19, 75-88.

DIOT M.F., 1991 - Apport et conservation sporo-pollinique dans les grottes : relation avec la fréquentation humaine et animale. Actes du colloque Expérimentation en archéologie : bilan et perspectives. Archéologie expérimentale, t. 2 : La Terre, édition Errance, Paris, 236-244.

ECHASSOUX A., D'ERRICO F., GIACOBINI G., DEL LUCCHESSE A., LUMLEY de H., PERPERE M., \& VICINO G., 1983 - La prima campagna di scavo alla Caverna delle Fate (Finale Ligure). Rivista Ingauna et Intermelia, 38, 50-53.

FAIRCHILD I.J., SMITH C.L., BAKER A., FULLER L., SPOTL C., MATTEY D., McDERMOTT F., \& E.I.M.F., 2006 - Modification and preservation of environmental signals in speleothems. Earth-Science Reviews, 75, 105-153.

FALGUÈRES C., YOKOYAMA Y., \& BIBRON R., 1990 - Electron spin resonance (ESR) of hominid-bearing deposits in the Caverna delle Fate, Liguria, Italy. Quaternary Research, 34, 121-128.

FALGUÈRES C., LAURENT M., AJAJA O., BAHAIN J.J., YOKOYAMA Y., \& HONG M.Y., 1991 - Datation par les méthodes U/Th et ESR de la grotte de la Baume Bonne (Alpes de HauteProvence). Actes du XII congrès U.I.S.P.P., Bratislava, Tchécoslovaquie, 98-107.

FALGUÈRES C., YOKOYAMA Y., SHEN G, BISCHOFF J.L., KU T.L., \& LUMLEY de H., 2004 - New U-series dates at the Caune de l'Arago, France. Journal of Archaeological Science, 31, 941-952. 
FARKH S., 2004 - Contribution à la datation des niveaux moustériens de la grotte de Karaïn (Antalya, Turquie) par les méthodes des séries de l'uranium $(U-T h)$ et la résonance de spin électronique (ESR) : méthodologie et paléoclimatologie. Thèse de doctorat, Muséum National d'Histoire Naturelle, Paris, 194 p.

FOLLIERI M., MAGRI D., \& SADORI L., 1988 - 250,000-year pollen record from Valle di Castiglione (Roma). Pollen et Spores, $30(3-4), 329-356$

FRENZEL B., 1964 - Zur Pollenanalyse von Lössen. Untersuchungen der Lössprofile von Oberfellabrunn und Stillfried (Niederösterreich). Eiszeitalter und Gegenwart, 15, 5-39.

GAGNEPAIN J., \& GAILLARD C., 1996 - La grotte-abri de la Baume Bonne : une séquence culturelle et chronostratigraphique de 300000 ans. Document final de synthèse de fouilles programmées. Programme triannuel : 1994-1995-1996, 93 p.

GAUTHIER A., 1984 - Contribution à l'étude palynologique du remplissage du Pléistocène moyen de la Caune de l'Arago (Tautavel, Pyrénées-Orientales). Analyse pollinique de la carotte $n^{\circ} 8$. Paléoclimatologie de l'ensemble stratigraphique V. Mémoire de DEA, MNHN Paris \& Université Paris 6, 56 p.

GAUTHIER A., 1992 - Paléoenvironnements du Pléistocène moyen dans le sud de la France. Apports et limites de l'analyse pollinique de trois sites préhistoriques: Caune de l'Arago, Orgnac 3, grotte du Lazaret. Thèse de doctorat, Muséum National d'Histoire Naturelle, Paris, $264 \mathrm{p}$.

GENTY D., \& QUINIF Y., 1996 - Annually laminated sequences in the internal structure of some Belgian stalagmites, importance for paleoclimatology. Journal of Sedimentary Research, 66, 275-288.

GENTY D., DIOT M.F., \& O'YL W., 2001 - Sources of pollen in stalagtite drip water in two caves in southwest France. Cave and Karst Science, 28, 59-66.

GENTY D., BLAMART D., OUAHRDI R., GILMOUR M., BAKER A., JOUZEL J., \& VAN-EXER S., 2003 - Precise dating of Dansgaard-Oeschger climate oscillations in western Europe from stalagmite data. Nature, 421, 833-838.

GIACOBINI G., LUMLEY de M.A., YOKOYAMA Y., \& NGUYEN H.V., 1984 - Neandertal child and adult remains from a Mousterian deposit in Northern Italy (Caverna delle Fate, Finale Ligure). Journal of Human Evolution, 13, 687-707.

GIRARD M., \& RENAULT-MISKOVSKY J., 1969 - Nouvelles techniques de préparation en palynologie appliquées à trois sédiments du Quaternaire final dans l'abri Cornille (Istres, Bouchesdu-Rhône). Bulletin de l'Association Française pour l'Etude du Ouaternaire, 4, 275-284.

HARMON R.S., THOMPSON P., SCHWARCZ H.P., \& FORD D.C., 1975 - Uranium series dating of speleothems. National Speleological Society Bulletin, 37, 21-33.

HOLLAND H.D., KIRSIPU T.V., HUEBNER J.S., \& OXBURGH U.M., 1964 - On some aspects of the chemical evolution of cave water. Journal of Geology, 72, 36-67.

JUVIGNÉ E., 1973 - Une méthode de séparation des pollens applicable aux sédiments minéraux. Annales de la Société Géologique de Belgique, 96 (2), 253-262.

KARATSORI E., 2003 - Environnement végétal de l'Homme fossile et climats en Ligurie pendant le dernier cycle climatique et le Postglaciaire. British Archaeological Reports International Series, Oxford, 1159, $250 \mathrm{p}$.

KARATSORI E., 2005 - Environnement de l'Homme de Néandertal en Ligurie au Pléistocène supérieur. Analyse pollinique de la $\mathrm{Ca}$ verna delle Fate (Finale Ligure, Italie). Comptes Rendus Palevol, 4, 395-404.

LARTIGOT A.S., 2002 - Contribution à l'étude palynologique de la grotte-abri de la Baume Bonne (Quinson, Alpes de Haute-Provence) : les ensembles stratigraphiques V, VIII et IX. Mémoire de DEA, Muséum National d'Histoire Naturelle, Paris, 73p.

LARTIGOT A.S. \& CROIZET S., 2005 - Analyses palynologiques de spéléothèmes en contexte archéologique. Mise en évidence d'œufs d'Helminthes. Symposium conjoint APLF-TMS-LSPG, Paris, p. 68.

LAURITZEN S.E., \& LUNDBERG J., 1999 - Speleothems and climate : a special issue of The Holocene. The Holocene, 9 (6), 643647.

LEBRETON V., 1997 - Etudes palynologiques des remplissages pléistocènes supérieurs de l'Abri Mochi (Baousse Rousse - Grimaldi - Ligurie italienne) et de la grotte de Kalamakia (Aréopolis Grèce). Mémoire de DEA, Muséum National d'Histoire Naturelle, Paris, 59 p.
LEBRETON V., \& DARLAS A., 2005 - Potentiel de l' analyse pollinique de stalagmites pour reconstituer l'environnement de l'Homme fossile : exemple du gisement de Kalamakia (Péloponnèse, Grèce). Symposium conjoint APLF-TMS-LSPG, Paris, p. 69.

LECERVOISIER B., 2003 - Etude stratigraphique, sédimentologique, micromorphologique et paléoclimatique de remplissages de grottes du Pléistocène supérieur ancien de l'Europe méditerranéenne: Sites moustériens du Boquete de Zafarraya (Andalousie), de Madonna dell'Arma (Ligurie) et de Kalamakia (Laconie, Péloponnèse). Thèse de doctorat, Muséum National d'Histoire Naturelle, Paris, $251 \mathrm{p}$.

LEROI-GOURHAN A., 1966 - L'analyse pollinique des coprolithes. Bulletin de la Société Préhistorique Française, 63 (5), 163 164

LEROI-GOURHAN A., 1967 - Pollens et datation de la grotte de la Vache (Ariège). Bulletin de la Société Préhistorique de l'Ariège, 22, 115-127.

LEROI-GOURHAN A., 1981 - Les pollens de la grotte de Comarque. Gallia Préhistoire, 24 (1), 87-88.

LI W.X., LUNDBERG J., DICKIN A.P., FORD D.C., SCHWARCZ H.P., McNUTT R., \& WILLIAMS D., 1989 High precision mass spectrometric uranium series dating of cave deposits and implications for palaeoclimate studies. Nature, 339, 534-536

LUMLEY de H., 1969 - La cabane acheuléenne dans la grotte du Lazaret (Nice). Mémoire de la Société Préhistorique Française, 7, $234 \mathrm{p}$.

LUMLEY de H., 1988 - La stratigraphie du remplissage de la grotte du Vallonnet. L'Anthropologie, 92 (2), 407-428.

LUMLEY de H., LUMLEY de M.A., MISKOVSKY J.C., \& RENAULT-MISKOVSKY J., 1976 - Grotte du Lazaret. In H. de Lumley \& L. Barral (ed.), Sites paléolithiques de la région de Nice et grottes de Grimaldi. Livret Guide de l'excursion Bl, IX ${ }^{\mathrm{e}}$ Congrès UISPP, Nice, 53-74.

LUMLEY de H., FOURNIER A., PARK Y.C., YOKOYAMA Y., \& DEMOUY A., 1984 - Stratigraphie du remplissage pléistocène moyen de la Caune de l'Arago à Tautavel. Etude de huit carottages effectués de 1981 à 1983. L'Anthropologie, 88 (1), 5-18.

LUMLEY de H., \& DARLAS A., 1994 - Grotte de Kalamakia (Aréopolis, Péloponnèse). Bulletin de Correspondance Hellénique, 118, 535-559.

LUMLEY-WOODYEAR de M.A., 1970 - Anténéandertaliens et néandertaliens du Bassin méditerranéen occidental européen. Thèse de doctorat d'Etat, Faculté des Sciences de Paris, 1116 p.

LUMLEY de M.A., 1981 - L'Homme de Tautavel. Critères morphologiques et stade évolutif. In H. de Lumley \& J. Labeyrie (ed.), $D a$ tations absolues et analyses isotopiques en Préhistoire-Méthodes et limites. CNRS, Prétirage, Paris, 259-264.

MARTIN P.S., \& SHARROCK F.W., 1964 - Pollen analysis of prehistoric human faeces : a new approach to ethnobotany. American antiquity, 30 (2), 168-180.

McGARRY S.F., \& CASELDINE C., 2004 - Speleothem Palynology: an undervalued tool in Quaternary studies. Quaternary Science Reviews, 23, 2389-2404.

MESSAGER E., 2002 - Contribution à l'étude paléoenvironnementale de la grotte de Karaïn E (Turquie) au travers de l'analyse palynologique des planchers stalagmitiques. Mémoire de DEA, Muséum National d'Histoire Naturelle, Paris, 72 p.

MESTOUR B., 1996 - Contribution à l'étude des mécanismes de mise en place et de l'évolution du remplissage de la Baume Bonne (Quinson, Alpes de Haute-Provence, France) : apports de l'analyse minéralogique et micromorphologique des dépôts phosphatés et carbonatés. Thèse de doctorat, Muséum National d'Histoire Naturelle, Paris, $241 \mathrm{p}$.

MOLLESON T.I., OAKLEY K.P., \& VOGEL J.C., 1972 - The antiquity of the human footprints of Tana della Basura. Journal of Human Evolution, 1, 467-471.

NAVARRO CAMACHO C., CARRION J.S., NAVARRO J., MUNUEA M., \& PRIETO A.R., 2000 - An experimental approach to the palynology of cave deposits. Journal of Quaternary Science, 15, 603-619.

NICOL-PICHARD S., 1985 - Analyse pollinique sur matériel carotté en site archéologique (Caune de l'Arago, Tautavel, PyrénéesOrientales). Comptes Rendus de l'Académie des Sciences, Série IIa, 300 (20), 1039-1044. 
OTTE M., YALCINKAYA I., TASKIRAN H., KOZLOWSKI J.K., BAR-YOSEF O., \& BAYON L., 1998 - Long-term technical evolution and human remains in the Anatolian Palaeolithic. Journal of Human Evolution, 33 (4), 413-431.

PERRENOUD C., 1993 - Origine et mise en place des paragenèses phosphatées de remplissages karstiques. Etude micromorphologique des sédiments de la Caune de l'Arago et de la Baume Bonne. Thèse de doctorat, Muséum National d'Histoire Naturelle, Paris, $185 \mathrm{p}$.

PONS A., \& REILLE M., 1988 - The Holocene- and Upper Pleistocene pollen record from Padul (Granada, Spain) : a new study. $\mathrm{Pa}$ laeogeography, Palaeoclimatology, Palaeoecology, 66, 243-263.

RAHARDJO A.T., 1984 - Contribution à l'étude palynologique du remplissage du Pléistocène moyen de la Caune de l'Arago à Tautavel (Pyrénées-Orientales). Analyse pollinique de la carotte $n^{\circ} 3$, paléoclimatologie de l'ensemble IV. Thèse de doctorat, Muséum National d'Histoire Naturelle, Paris \& Université Paris 6, 143p.

RAHARDJO A.T., \& RENAULT-MISKOVSKY J., 1984 - Palaeoclimatological study of middle Pleistocene sediment based on pollen analysis at the Arago site, Southern France. Buletin Jurusan, 12, 23-28.

RENAULT-MISKOVSKY J., 1976 - La végétation au Pléistocène moyen en Languedoc méditerranéen. In $\mathrm{H}$. de Lumley (ed.), La Préhistoire Française. Editions du CNRS, Paris, 1, 486-488.

RENAULT-MISKOVSKY J., 1980 - Le Paléoenvironnement de l'Homme de Tautavel (Caune de l'Arago, Tautavel, PyrénéesOrientales): la faune et la flore en Roussillon au Pléistocène moyen. Comptes Rendus de l'Académie des Sciences, Série IIa, 290, 747-750.

RENAULT-MISKOVSKY J., 1981 - Etude palynologique du remplissage de la Caune de l'Arago à Tautavel. Signification chronologique, paléoclimatique et palethnographique des flores. In $\mathrm{H}$. de Lumley \& J. Labeyrie (ed.), Datations absolues et analyses isotopiques en Préhistoire-Méthodes et limites. CNRS, Prétirage, Paris, 253-258

RENAULT-MISKOVSKY J., 1995 - Middle Pleistocene vegetation and paleoclimatology in the French Mediterranean Basin. Actas, $\mathbf{1}$, 74-97.

RENAULT-MISKOVSKY J., \& GIRARD M., 1976 - La végétation au Pléistocène inférieur en Provence. In H. de Lumley (ed.), La Préhistoire Française. Editions du CNRS, Paris, 1, 468-471.

RENAULT-MISKOVSKY J., \& GIRARD M., 1978 - Analyse pollinique du remplissage pleistocène inférieur et moyen de la Grotte du Vallonnet (Roquebrune-Cap-Martin, Alpes-Maritimes). Géologie méditerranéenne, 4, 385-402.

RENAULT-MISKOVSKY J., \& GIRARD M., 1988 - Palynologie du remplissage de la Grotte du Vallonnet (Roquebrune-Cap-Martin, Alpes-Maritimes). Nouvelles données chronologiques et paléoclimatiques. L'Anthropologie, 92 (2), 437-448.

RENAULT-MISKOVSKY J., \& TEXIER P.J., 1980 - Intérêt de l'analyse pollinique détaillée dans les concrétions de grottes. Application à l'Abri Pié-Lombard (Tourettes-sur-Loup, AlpesMaritimes). Bulletin de l'Association Française pour l'Etude du Quaternaire, 3, 129-134.

ROUSSEAU L., BEAUCHAMP J., FALGUÈRES C., EMBLANCH C., GENTY D., BAHAIN J.J., \& BLAMART D. 2005 - Apport des marqueurs isotopiques et biogéochimiques dans la reconstitution du paléoenvironnement de la grotte du Lazaret (Nice, Alpes-Maritimes) au cours du Pléistocène supérieur (stade isotopique 5). Comptes Rendus Geoscience, 337, 1348-1354.

ROUSSEAU L., POZZI J.P., FALGUÈRES C., BAHAIN J.J., \& BEAUCHAMP J., 2006 - Non marine geological studies (speleothem, travertine) providing information pertinent to palaeoclimatology studies for old geological periods of the Quaternary. Colloque international Q5 Le Quaternaire, limites et spécificités, Paris, p. 32

SHEN G., 1985 - Datations des planchers stalagmitiques de sites acheuléens en Europe par les méthodes des déséquilibres des familles de l'Uranium et contribution méthodologique. Thèse de doctorat d'Etat, Université Paris 6, 162 p.

SHOPOV Y.Y., \& DERMENDJIEV V., 1990 - Microzonality of luminescence of cave flowstones as a new indirect index of solar activity. Comptes Rendus de l'Académie Bulgare des Sciences, $\mathbf{4 3}$, 9-12.

SITTLER C., 1955 - Méthodes techniques physico-chimiques de préparation des sédiments en vue de leur analyse pollinique. Revue de l'Institut français du Pétrole, 10 (2), 103-114.

SMART P.L., \& FRIEDERICH H., 1986 - Water movement and storage in the unsaturated zone of a maturely karstified aquifer, Mendip Hills, England. Proceedings of the Conference on Environmental Problems in Karst Terrains and their Solutions. National Water Wells Association, Bowling Green, Kentucky, 57-87.

SMITHSON P.A., 1982 - Temperature variations in Creswell Crags (near Worksop). East Midlands Geographer, 8, 51-64.

TEXIER P.J., 1974 - L'industrie moustérienne de 1'Abri Pié-Lombard (Tourettes-sur-Loup, Alpes-Maritimes). Bulletin de la Société Préhistorique Française, 6, 429-448.

TZEDAKIS P.C., 1993 - Long-term tree populations in northwest Greece through multiple Quaternary climatic cycles. Nature, 364, 437-440.

TZEDAKIS P.C., 1999 - The last climatic cycle at Kopais, central Greece. Journal of the Geological Society, 156, 425-434.

TZEDAKIS P.C., HOOGHIEMSTRA H., \& PALIKE H., 2006 The last 1.35 million years at Tenaghi Philippon : revised chronostratigraphy and long-term vegetation trends. Ouaternary Science Reviews, 25, 3416-3430.

VALLADAS H., CHADELLE J.P., GENESTE J.M., JORON J.L., MEIGNEN L., \& TEXIER P.J., 1987 - Datations par thermoluminescence de gisements moustériens du sud de la France. L'Anthropologie, 91 (1), 211-222.

VALLADAS H., GENESTE J.M., MEIGNEN L., \& TEXIER P.J., 1988 - Datations par thermoluminescence de gisements moustériens du sud de la France. In H.P. Schwarcz (ed.), L'Homme de Néandertal. La chronologie. ERAUL, Liège, 28 (1), 121-123.

VAN CAMPO M., 1950 - Une méthode de préparation très rapide des tourbes en vue de leur analyse pollinique. Bulletin de la Société Botanique de France, 97, 7-9.

VAN CAMPO M., \& LEROI-GOURHAN A., 1956 - Note préliminaire à l'étude des pollens fossiles de différents niveaux des grottes d'Arcy-sur-Cure. Bulletin du Muséum National d'Histoire Naturelle, 28 (3), 326-330.

VOINCHET P., \& DARLAS A., 1998 - Dating stratigraphic levels in Kalamakia Cave (Greece). Arkeos, 5, 45-51.

VON POST L., 1916 - Om skogsträdpollen i sydvenska torfmosslagerföljder. Geologiska Föreningens i Stockholm Förhandlingar, 38, 384 .

WIJMSTRA T.A., 1969 - Palynology of the first 30 meters of a $120 \mathrm{~m}$ deep section in northern Greece. Acta Botanica Neerlandica, 18 (4), 512-527.

WIJMSTRA T.A., \& SMIT A., 1976 - Palynology of the middle part (30-78 meters) of the $120 \mathrm{~m}$ deep section in northern Greece (Macedonia). Acta Botanica Neerlandica, 25 (4), 297-312

YALCINKAYA I., 1988 - Résultats récents des fouilles à Karaïn en Anatolie. In J.K. Kozlowski (ed.), L'Homme de Neandertal : la mutation. ERAUL, Liège, 8, 257-271.

YALCINKAYA I., 1998 - La grotte de Karaïn : généralité dans le contexte anatolien. In M. Otte (ed.), Préhistoire d'Anatolie, Genèse entre deux mondes. ERAUL, Liège, 85, 453-461.

YOKOYAMA Y., QUAEGEBEUR J.P., BIBRON R., LEGER C., NGUYEN H.V., \& POUPEAU G., 1982 - Datation du site de l'Homme de Tautavel par la résonance de spin électronique (ESR). Comptes Rendus de l'Académie des Sciences, Série IIa, 294, 759. 764.

YOKOYAMA Y., SHEN G., \& VAN NGUYEN H., 1985 - Dating of stalagmitic carbonates and bones of the Bàsura Cave at Toirano (Liguria, Italy) by the U-Th and U-Pa methods using alpha- and gamma- ray spectrometries. Revue d'Etudes Ligures, 51 (4), 373 378

YOKOYAMA Y., BIBRON R., \& FALGUÈRES C., 1988 - Datations absolues des planchers stalagmitiques de la grotte du Vallonnet à Roquebrune-Cap-Martin (Alpes-Maritimes) France, par la résonance de spin électronique (ESR). L'Anthropologie, 92 (2), 429-436 